\title{
The Generalized Incomplete Gamma Functions
}

\section{Didem Aşçıı̆̆lu}

\author{
Submitted to the \\ Institute of Graduate Studies and Research \\ in partial fulfillment of the requirements for the Degree of
}

\author{
Master of Science \\ in \\ Mathematics
}

Eastern Mediterranean University

September 2015

Gazimağusa, North Cyprus 
Approval of the Institute of Graduate Studies and Research

\section{Prof. Dr. Serhan Çiftçioğlu} Acting Director

I certify that this thesis satisfies the requirements as a thesis for the degree of Master of Science in Mathematics.

Prof. Dr. Nazım I. Mahmudov

Chair, Department of Mathematics

We certify that we have read this thesis and that in our opinion it is fully adequate in scope and quality as a thesis for the degree of Master of Science Mathematics.

Asst. Prof. Dr. Mustafa Kara

Supervisor

Examining Committee

1. Prof. Dr. Nazım I. Mahmudov

2. Asst. Prof. Dr. Mustafa Kara

3. Asst. Prof. Dr. Nidai Şemi 


\begin{abstract}
Engineering and physics demand a through knowledge of applied mathematics and a good understanding of special functions. These functions commonly arise in such areas of applications as heat conduction, communication systems, electro-optics, approximation theory, probability theory, and electric circuit theory, among others. The subject of special functions is quite rich and expanding continuously with the emergence of new problems in the areas of applications in engineering and applied sciences. We investigate generalized gamma function, digamma function, the generalized incomplete gamma function, extended beta function. Also, some properties of these functions are taken into hand.
\end{abstract}

Keywords: Approximation, Circuit, Gamma, Beta, Digamma 


\section{ÖZ}

Mühendislik ve fizik, uygulamalı matematiğin derinlemesine bilinmesini ve özel fonksiyonların iyi anlaşılmasını istemektedir. Bu fonksiyonlar genellikle 1sı iletimi, iletişim sistemleri, elektro-optik, yaklaşıklık teorisi, olasıklık teorisi, elektrik aksam teorisi ve diğerleri alanlarında uygulama bulur. Özel fonksiyonlar konusu oldukça zengin ve genişlemeye açık bir alan bunun sebebi ise mühendislik ve uygulamalı bilimler alanlarındaki yeni problem doğuşlarıdır. Biz genelleştirilmiş gama fonksiyonu, digamma fonksiyonu, genişletilmiş beta fonksiyonu ve bu fonksiyonların bazı özelliklerini inceledik.

Anahtar Kelimeler: Yaklaşıklık, Aksam, Gamma, Beta, Digamma 
To My Family 


\section{ACKNOWLEDGEMENT}

Firstly, I would like to thank my supervisor, Asst. Prof. Dr. Mustafa Kara, for his patience, motivation, enthusiasm, knowledge and giving me the opportunity to work with him. His guidance helped me in all the time of research and writing of this thesis.

Then, I would like to thank Prof. Dr. Nazım I. Mahmudov, Assoc. Prof. Dr. Sonuç Zorlu, Asst. Prof. Dr. Nidai Şemi , Prof. Dr. Aghamirza Bashirov for their support during my MS education.

Also, I would like to thank my family for their love, care and support during my life. 


\section{TABLE OF CONTENTS}

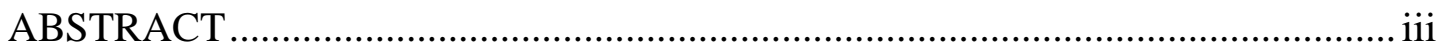

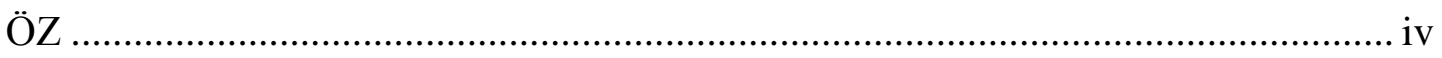

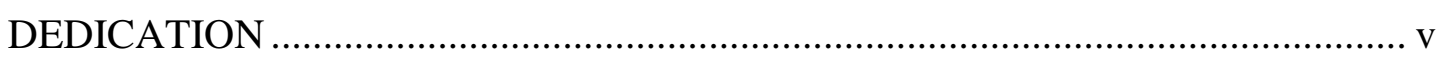

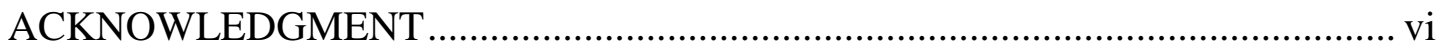

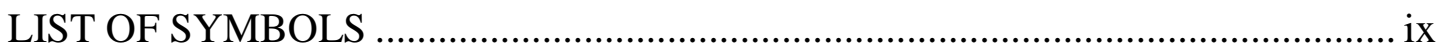

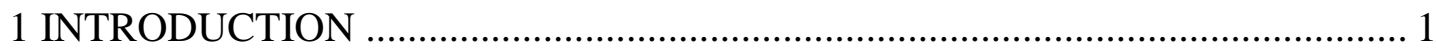

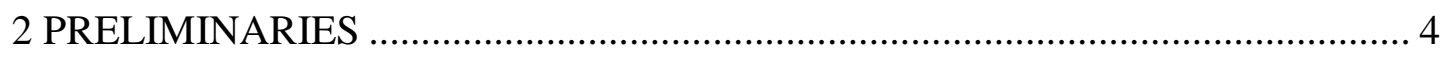

3 THE GAMMA AND BETA FUNCTIONS ................................................... 8

3.1 Definition of the Gamma Function ........................................................... 8

3.2 Reccurence Relation of Gamma Function ................................................ 8

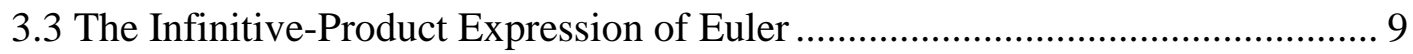

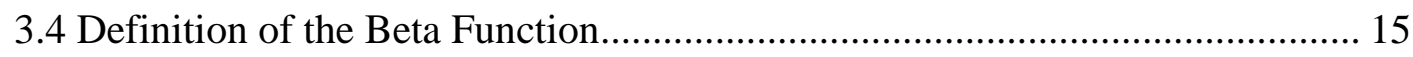

3.5 The Infinitive Product Expression of Beta Function ................................... 17

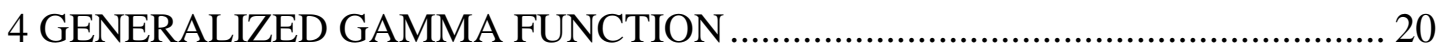

4.1 Definition of the Generalized Gamma Functions .......................................... 20

4.2 Properties of the Generalized Gamma Functions........................................... 21

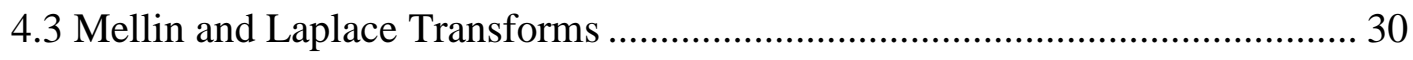

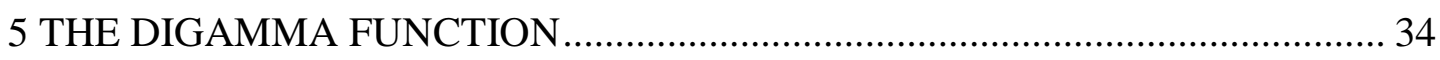

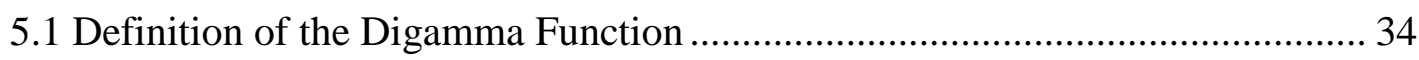

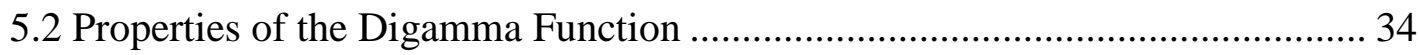

5.3 Generalization of the Psi (Digamma) Function .............................................. 39

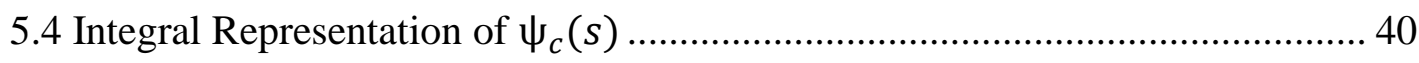

5.5 Properties of the Generalized psi Function ................................................. 46 
6 THE GENERALIZED INCOMPLETE GAMMA FUNCTION ........................... 50

6.1 The Incomplete Gamma Functions …........................................................ 50

6.2 Definition of the Generalized Incomplete Gamma Functions ......................... 50

6.3 Properties of the Incomplete Generalized Gamma Functions.......................... 51

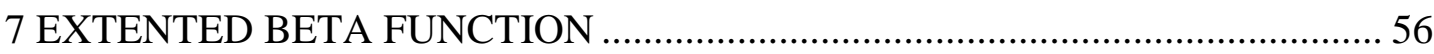

7.1 Definition of the Extended Beta Function ...................................................... 56

7.2 Properties of the Extended Beta Function....................................................... 56

7.3 Integral Representations of the Extended Beta Function................................. 59

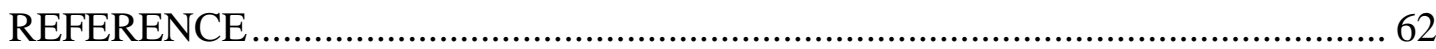




\section{LIST OF SYMBOLS}

\begin{tabular}{ll}
$\Gamma(x, y)$ & gamma function \\
$\gamma$ & the Euler - Mascheroni Constant \\
$B(x, y)$ & Beta function \\
$B(x, y ; c)$ & extended beta function \\
$M$ & Mellin \\
$M-1$ & inverse of the mellin \\
$\mathcal{L}$ & Laplace \\
$\Gamma(s, x)$ & the upper incomplete gamma function \\
$\gamma(s, x)$ & the lower incomplete gamma function \\
$K_{y}$ & macdonald function \\
$I_{y}$ & the set of real numbers \\
$\Gamma_{c}(x)$ & modified Bessel function \\
$W_{c}$ & generalized gamma function \\
$\psi_{c}$ & digamma induced by c \\
& Mellin transform \\
\hline &
\end{tabular}




\section{Chapter 1}

\section{INTRODUCTION}

Between 1927 and 1930, Euler introduced an analytic function which has the property to interparticle the fuctorial whenever the argument of the function is an integer. But, in 1930, Euler introduced the following functions.

$$
\Gamma(x)=\int_{0}^{1}(-\log (t))^{x-1} d t \quad(x>0)
$$

After some easy process this definitions take more used forms as

$$
\Gamma(x)=\int_{0}^{\infty} t^{x-1} e^{-t} d t
$$

In [5] L. S. Gradshteyn and L. M. Ryzhink, introduced the generalized gamma function as the following.

$$
\Gamma_{c}(s)=\int_{0}^{\infty} t^{s-1} e^{-t-c t^{-1}} d t \quad(\operatorname{Re}(c)>0 ; c=0, \operatorname{Re}(s)>0)
$$

First derivative of $\log \Gamma(s)$ is called digamma $f$ and denoted by

$$
\psi(s)=\frac{\Gamma^{\prime}(s)}{\Gamma(s)}
$$

In [1] M. Aslam Chaudhry and Syed M. Zubair gave the definition of the digamma function as the following

$$
\psi_{c}(s)=\frac{d}{d s}\left\{\ln \left(\Gamma_{c}(s)\right)\right\}
$$


In [1] M. Aslam Chaudhry and Syed M. Zubair, introduced the generalized incomplete gamma function as the following

$$
\begin{aligned}
& \gamma(s, x)=\int_{0}^{x} t^{s-1} e^{-t} d t \quad(s=\sigma+i r ; \sigma>0, \quad|\arg (s)|<\pi), \\
& \Gamma(s, x)=\int_{x}^{\infty} t^{s-1} e^{-t} d t \quad(|\arg (s)|<\pi)
\end{aligned}
$$

Also, [1] M. Aslam Chaudhry, Syed M. Zubair and [15] Asghar Qadir, M. Rafique introduced the integral representation of the extended beta function

$$
B(x, y ; c)=\int_{0}^{1} t^{x-1}(1-t)^{y-1} e^{-\frac{c}{t(1-t)}} d t
$$

My thesis contains seven chapters. The name of this chapters are listed below.

- Introduction

- Preliminary

- The Gamma and Beta Functions

- Generalized Gamma Function

- The Digamma Function

- The Generalized Incomplete Gamma Functions

- Extented Beta function

Chapter 2, contains some important definitions and theorems (The Euler Mascheroni Constant, Fubini's, Laplace Transform, Mellin Transform, Macdonald Function, Wittaker Function, Hölder's İnequality, Fundamental). 
In Chapter 3, the following studied

- Definition of Beta and Gamma Functions

- Reccurence relation of gamma function

- the infinitive-product expression of euler and beta function

Essentially, Chapter 4, 5, 6 and 7 contains definitions and properties of

- Generalized Gamma Function

- The Digamma Function

- The Generalized Incomplete Gamma Function

- Extended Beta Function 


\section{Chapter 2}

\section{PRELIMINARIES}

Theorem 2.0.1 [18] (The Euler Mascheroni Constant) Euler Mascheroni constant, defined by

$$
\gamma=\lim _{n \rightarrow \infty}\left(\sum_{m=1}^{n} \frac{1}{m}-\log n\right)
$$

which is approximately equal to $0.5772 \ldots$

Theorem 2.0.2 [4](Fubini's Theorem) Let $\mu$ be a measure on $T$ and $v$ a measure on $Z$.

$i$. Then $\mu \times v$ is regular measure on $T \times Z$, even if $\mu$ and $v$ are not regular.

ii. If $A \subset T$ is $\mu$-measurable and $B \subset Z$ is $v-$ measurable, then $A \times B$ is $(\mu \times v)-$ measurable and $(\mu \times v)(A \times B)=\mu(A) v(B)$

iii. If $S \subset T \times Z$ is a-finite with respect to $\mu \times v$, then $S_{z} \equiv\{t \mid(t, z) \in S\}$ is $\mu$-measurable for $v$ a.e.y, $S_{t} \equiv\{z \mid(t, z) \in S\}$ is $v$-measurable for $\mu$-integrable. Moreover,

$$
(\mu \times v)(s)=\int_{Z} \mu\left(S_{z}\right) d v(z)=\int_{T} v\left(S_{t}\right) d \mu(t)
$$

iv. If $f$ is $(\mu \times v)$-integrable and $f$ is a-finite with respect to $\mu \times v$ (in particular, 
if $f$ is $(\mu \times v)$-summable), then the mapping $z \longmapsto \int_{T}(t, z) d \mu(t)$ is $v$-integrable

$$
z \longmapsto \int_{T} f(t, z) d v(t) \text { is } v \text {-integrable }
$$

the mapping

$$
t \longmapsto \int_{Z} f(t, z) d v(z) \text { is } \mu \text {-integrable. }
$$

and

$$
\begin{aligned}
\int_{T \times Z} f d(\mu \times v) & =\int_{Z}\left[\int_{T} f(t, z) d \mu(t)\right] d v(z) \\
& =\int_{T}\left[\int_{Z} f(t, z) d v(z)\right] d \mu(t)
\end{aligned}
$$

Theorem 2.0.3 [10] (Laplace Transform) Suppose that $f(a)$ is a piecewise continuous on $[0, \infty)$ and it is of exponential order $\alpha$. Then the $\mathscr{L}$ - transform of the function $f(a)$ exists for all $s>\alpha$ and real numbers $t>0$, which is given by:

$$
\mathscr{L}(f(t))=F(s)=\int_{0}^{\infty} e^{-s t} f(t) d t
$$

Definition 2.0.4 [10] (Mellin Transform) The Mellin transform of a function $f(t) d e$ fined by

$$
\{M f\}(s)=\varphi(s)=\int_{0}^{\infty} t^{s-1} f(t) d t
$$

and the inverse of the Mellin Transform defined as the following

$$
\left\{M^{-1} \varphi\right\}(t)=f(t)=\frac{1}{2 \pi i} \int_{u-i \infty}^{u+i \infty} t^{-s} \varphi(s) d s
$$


Theorem 2.0.5 [5] (Macdonald Function) Modified Bessel functions of the third kind or Macdonald functions defined by

$$
K_{y}(z)=\frac{\pi}{2} \frac{I_{-y}(z)-I_{y}(z)}{\sin y \pi}
$$

where $I_{y}(z)$ is modified Bessel function of first kind [6] given by

$$
I_{y}(z)=\sum_{n=0}^{\infty} \frac{\left(\frac{z}{2}\right)^{y+2 n}}{\Gamma(y+n+1) n !}
$$

Theorem 2.0.6 [17] (Whittaker Function)

$$
\int_{0}^{\infty} t^{\mu-\frac{1}{2}} e^{-\alpha x} K_{2 y}(2 \beta \sqrt{t}) d t
$$

The inverse Laplace Transform of the product

$$
\frac{\Gamma\left(\mu+y+\frac{1}{2}\right) \Gamma\left(\mu-y+\frac{1}{2}\right)}{2 \beta} e^{\frac{\beta^{2}}{2 \alpha}} \alpha^{-\mu} W_{-\mu, v}\left(\frac{\beta^{2}}{\alpha}\right)
$$

Theorem 2.0.7 [17](Hölder's inequality)

Let $\frac{1}{n}+\frac{1}{m}=1$ with $n, m>1$. Then Hölder's inequality for integrals states that

$$
\int_{a}^{b}|f(x) g(x)| d x \leq\left[\int_{a}^{b}|f(x)|^{n} d x\right]^{\frac{1}{n}}\left[\int_{a}^{b}|g(x)|^{m} d x\right]^{\frac{1}{m}}
$$

Theorem 2.0.8 [21] (The Fundamental Theorem of Calculus) Suppose that the function $f$ is a continuous on an interval I containing the point a, 
Part1: Let the function $F$ be defined on $I$ by

$$
F(x)=\int_{a}^{x} f(t) d t
$$

Then $F$ is differentiable on $I$ and $F^{\prime}(x)=f(x)$ there. Thus, $F$ is an antiderivative of on $I$ :

$$
\frac{d}{d x}\left(\int_{a}^{x} f(t) d t\right)=f(x)
$$

Part2: If $G(x)$ is any antiderivative of $f(x)$ on $I$, so that $G^{\prime}(x)=f(x)$ on $I$, then any $b$ in $I$ we have

$$
\int_{a}^{b} f(t) d t=G(b)-G(a)
$$




\section{Chapter 3}

\section{THE GAMMA AND BETA FUNCTIONS}

This chapter contains basic definitions and properties of the Gamma and Beta functions.

\subsection{Definition of the Gamma Function}

For complex numbers with positive real part defined as the following

$$
\Gamma(s)=\int_{0}^{\infty} t^{s-1} e^{-t} d t
$$

The equation below is stated by using Mellin transform in gamma function

$$
\Gamma(s)=\left\langle t_{+}^{s-1}, e^{-t}\right\rangle=\int_{0}^{\infty} t^{s-1} e^{-t} d t
$$

\subsection{Reccurence Relation of Gamma Function}

We have obviously $\Gamma(1)=\int_{0}^{\infty} e^{-t} d t=1$ and for $x>0$, an integration by parts yields.

$$
\begin{gathered}
\Gamma(x)=\left[e^{-t} \frac{t^{x}}{x}\right]_{0}^{\infty}+\int_{0}^{\infty} \frac{t^{x}}{x} e^{-t} d t=x^{-1} \int_{0}^{\infty} t^{x} e^{-t} d t \\
=x^{-1} \Gamma(x+1) .
\end{gathered}
$$


Hence,

$$
\Gamma(x+1)=x \Gamma(x) .
$$

For $\forall n \in \mathbb{Z}^{+}$, we have

$$
\begin{aligned}
\Gamma(n+1) & =n \Gamma(n) \\
& =n(n-1) \Gamma(n-1) \\
& =n(n-1)(n-2) \Gamma(n-2) \\
& =n(n-1) \ldots \ldots .2 .1 \Gamma(1) \\
& =n !
\end{aligned}
$$

where by convention, $0 !=1$

\subsection{The Infinite-Product Expression of Euler}

Lemma 3.3.1 [9] If $0 \leq z<1$, then

$$
1+z \leq e^{z} \leq \frac{1}{1-z}
$$

Proof. From the series expression of $e^{z}$ and $(1-z)^{-1}$, we have

$$
1+z \leq e^{z} \text { and } \quad e^{z} \leq \frac{1}{1-z}
$$

Since,

$$
1+z \leq e^{z}=1+z+\sum_{k=2}^{\infty} \frac{z^{k}}{k !} . \quad \text { and } \quad e^{z} \leq \frac{1}{1-z}=\sum_{k=0}^{\infty} z^{k}
$$

This concludes the proof. 
Lemma 3.3.2 [9] If $0 \leq z<1$ and $n \in \mathbb{Z}^{+}$, then

$$
(1-z)^{n} \geqslant 1-n z
$$

Proof. Using the mathematical induction method

i. For $n=1 \Longrightarrow 1-z=1-z$

ii. Assume that $(1-z)^{k} \geqslant(1-k z)$ is true, $\left(k \in \mathbb{Z}^{+}\right)$

iii. For $n=k+1$

$$
\begin{aligned}
(1-z)^{k+1} & =(1-z)^{k}(1-z) \\
& \geqslant(1-k z)(1-z) \\
& =1-(k+1) z+k z^{2} \\
& \geqslant 1-(k+1) z,\left(k \in \mathbb{Z}^{+}\right) .
\end{aligned}
$$

Hence, the results holds for all $n \in \mathbb{Z}^{+}$by mathematical induction.

Lemma 3.3.3 [9] If $0 \leqslant t<n$ and $n \in \mathbb{Z}^{+}$, then

$$
0 \leqslant e^{-t}-\left(1-\frac{t}{n}\right)^{n} \leqslant \frac{t^{2}}{n} e^{-t}
$$

Proof. Using Lemma 3.3.1 with $z=\frac{t}{n}(0 \leqslant z<1)$ we get

$$
1+\frac{t}{n} \leqslant \exp \left(\frac{t}{n}\right) \leqslant\left(1-\frac{t}{n}\right)^{-1}
$$




$$
\left(1+\frac{t}{n}\right)^{n} \leqslant e^{t} \leqslant\left(1-\frac{t}{n}\right)^{-n}
$$

and

$$
\left(1+\frac{t}{n}\right)^{-n} \geqslant e^{-t} \geqslant\left(1-\frac{t}{n}\right)^{n}
$$

so that

$$
e^{-t}-\left(1-\frac{t}{n}\right)^{n} \geqslant 0
$$

on the other hand

$$
\begin{aligned}
e^{-t}-\left(1-\frac{t}{n}\right)^{n} & =e^{-t}\left[1-e^{t}\left(1-\frac{t}{n}\right)^{n}\right] \\
& \leqslant e^{-t}\left[1-\left(1-\frac{t^{2}}{n}\right)^{n}\right]
\end{aligned}
$$

Apply Lemma 3.3.2 with $z=\frac{t^{2}}{n^{2}}$

$$
\left(1-\frac{t^{2}}{n^{2}}\right)^{n} \geqslant 1-\frac{t^{2}}{n}
$$

and by (3.3.3), we have

$$
e^{-t}-\left(1-\frac{t}{n}\right)^{n} \leqslant e^{-t}\left[1-1+\frac{t^{2}}{n^{2}}\right]=\frac{t^{2}}{n} e^{-t}
$$

The result follows from (3.3.1) and (3.3.2)

Lemma 3.3.4 [9] If $n \in \mathbb{Z}$ and $\operatorname{Re}(z)>0$, then

$$
\Gamma(z)=\lim _{n \longrightarrow \infty} \int_{0}^{n}\left(1-\frac{t}{n}\right)^{n} t^{z-1} d t .
$$


Proof. Let

$$
P_{n}(z)=\int_{0}^{n}\left(1-\frac{t}{n}\right)^{n} t^{z-1} d t
$$

We need to prove that, $P_{n}(z) \rightarrow \Gamma(z)$ when $n \longrightarrow \infty$,

$$
\begin{aligned}
\int_{0}^{\infty} e^{-t} t^{z-1} d t-P_{n}(z) & =\left[\int_{0}^{\infty} e^{-t} t^{z-1} d t-\int_{0}^{n}\left(1-\frac{t}{n}\right)^{n} t^{z-1} d t\right] \\
& =\int_{0}^{n}\left\{e^{-t}-\left(1-\frac{t}{n}\right)^{n}\right\} t^{z-1} d t+\int_{0}^{\infty} e^{-t} t^{z-1} d t
\end{aligned}
$$

Obviously, the limit of the second term is zero, when $n \longrightarrow \infty$ and the limit of the first term is also zero, since

$$
\left|\int_{0}^{n}\{\underbrace{e^{-t}-\left(1-\frac{t}{n}\right)^{n}}_{\leqslant \frac{t^{2} e^{-t}}{n}}\} t^{z-1} d t\right| \leqslant \int_{0}^{n} \frac{1}{n} e^{-t} t^{z+1} d t
$$

$$
<\int_{0}^{n} \frac{1}{n} e^{-t} t^{x+1} d t \longrightarrow 0
$$

Hence $P_{n}(z) \rightarrow \Gamma(z)$.

Lemma 3.3.5 [9] If $n \in \mathbb{Z}$ and $\operatorname{Re}(z)>0$, then

$$
\Gamma(z)=\lim _{n \rightarrow \infty} \frac{n ! n^{z}}{z(z+1) \ldots(z+n)} .
$$


Proof. For $\operatorname{Re}(z)>0$, substitute $t=n \tau$ and $d t=n d \tau$ in Lemma 3.3.4

$$
P_{n}(z)=\int_{0}^{1}(1-\tau)^{n}(n \tau)^{z-1} n d \tau
$$

If we choose $u=(1-\tau)^{n}$ and $d v=\tau^{z-1}$ in 3.3.3, then apply, integration by parts n-times, we get

$$
\begin{aligned}
P_{n}(z) & =n^{z}\left\{\left[(1-\tau)^{n} \frac{\tau^{z}}{z}\right]_{0}^{1}+n \int_{0}^{1} \frac{\tau^{z}}{z}(1-\tau)^{n-1} d \tau\right\} \\
& =\frac{n^{z} n(n-1) \ldots 2.1}{z(z+1) \ldots(z+n-1)} \int_{0}^{1} \tau^{z+n-1} d \tau \\
& =\frac{1.2 \ldots n}{z(z+1) \ldots(z+n)} n^{z} .
\end{aligned}
$$

So,

$$
\Gamma(z)=\lim _{n \rightarrow \infty} \frac{1.2 \ldots n}{z(z+1) \ldots(z+n)} n^{z}
$$

Since $P_{n}(z) \rightarrow \Gamma(z)$ when $n \rightarrow \infty$.

Lemma 3.3.6 [9] If $n \in \mathbb{Z}$ and $\operatorname{Re}(z)>0$, then

$$
\Gamma(z)=\frac{1}{z} \prod_{n=1}^{\infty}\left[\left(1+\frac{z}{n}\right)^{-1}\left(1+\frac{1}{n}\right)^{-z}\right]
$$

Proof. Using Lemma 3.3.5 and $\lim _{n \rightarrow \infty} \frac{n}{z+n}=1$, we get

$$
\Gamma(z)=\lim _{n \rightarrow \infty} \frac{1.2 \ldots(n-1)}{z(z+1) \ldots(z+n-1)} n^{z}
$$


$n^{z}$ and $(z+1) \ldots(z+n-1)$ can be written as

$$
n^{z}=\prod_{m=1}^{n-1}\left(1+\frac{1}{m}\right)^{z}
$$

and

$$
(z+1) \ldots(z+n-1)=\frac{1}{z} \prod_{m=1}^{n-1}\left(1+\frac{z}{m}\right)^{-1} .
$$

Hence, we get

$$
\Gamma(z)=\frac{1}{z} \prod_{n=1}^{\infty}\left[\left(1+\frac{z}{n}\right)^{-1}\left(1+\frac{1}{n}\right)^{-z}\right]
$$

This gives the desired result.

Theorem 3.3.7 [9] (Weierstrass Infinitive Product) If $\operatorname{Re}(z)>0$, then

$$
\frac{1}{\Gamma(z)}=z e^{\gamma z} \prod_{n=1}^{\infty}\left\{\left(1+\frac{z}{n}\right) e^{-\frac{z}{n}}\right\}
$$

where

$$
\begin{gathered}
\gamma=\lim _{n \rightarrow \infty}\left(\sum_{k=1}^{n} \frac{1}{k}-\log n\right) \\
=0.57721566490153286060651 \ldots
\end{gathered}
$$

and denotes Euler constant. 
Proof. In equation 3.3.4 $n^{z}$ can be as

$$
n^{z}=\exp \left\{z\left[\ln n-\sum_{m=1}^{n} \frac{1}{m}\right]\right\} \prod_{m=1}^{n} e^{\frac{z}{m}}
$$

if equation 3.3.6 is substituted by 3.3.4 then, we get

$$
\begin{aligned}
& \lim _{n \rightarrow \infty} \frac{1.2 \ldots n}{z(z+1) \ldots(z+n)} \exp \left\{z\left[\ln n-\sum_{m=1}^{n} \frac{1}{m}\right]\right\} \prod_{m=1}^{n} e^{\frac{z}{m}} \\
& \frac{1}{\Gamma(z)}=\frac{z(z+1) \ldots(z+n)}{1.2 \ldots n} \exp \left\{z\left[\sum_{m=1}^{n} \frac{1}{m}-\ln n\right]\right\} \prod_{m=1}^{n} e^{-\frac{z}{m}} .
\end{aligned}
$$

Hence

$$
\frac{1}{\Gamma(z)}=z e^{\gamma z} \prod_{n=1}^{\infty}\left\{\left(1+\frac{z}{n}\right) e^{-\frac{z}{n}}\right\}
$$

where

$$
\gamma=\lim _{n \rightarrow \infty}\left(\sum_{m=1}^{n} \frac{1}{m}-\ln n\right)
$$

is known.

\subsection{Definition of the Beta Function}

Definition 3.4.1 [2] (Beta Function) Euler integral of the first kind or Beta integral is

a function of two complex variables defined by

$$
B(s, q)=\int_{0}^{1} t^{s-1}(1-t)^{q-1} d t \quad(\operatorname{Re}(s)>0 ; \operatorname{Re}(q)>0)
$$


Theorem 3.4.2 [1] For $s, q \notin \mathbb{Z}_{0}=\{0,-1,-2, \ldots\}$,

$$
B(s, q)=\frac{\Gamma(s) \Gamma(q)}{\Gamma(s+q)}\left(s, q \notin Z_{0}^{-}=\{0,-1,-2, \ldots\}\right)
$$

Proof. Putting $t=\sin ^{2} \theta$ and $d t=2 \sin \theta \cos \theta d \theta$ in (3.4.1), we get

$$
\begin{aligned}
B(s, q) & =2 \int_{0}^{\frac{\pi}{2}} \sin ^{2 s-1} \theta\left(1-\sin ^{2} \theta\right)^{q-1} \cos \theta d \theta \\
& =2 \int_{0}^{\frac{\pi}{2}} \sin ^{2 s-1} \theta \cos ^{2 q-1} d \theta \quad(\operatorname{Re}(s)>0 ; \operatorname{Re}(q)>0)
\end{aligned}
$$

On the other hand

$$
\Gamma(s) \Gamma(q)=\int_{0}^{\infty} e^{-t} t^{s-1} d t \int_{0}^{\infty} e^{-t} v^{q-1} d v
$$

substitution $t=w^{2}, v=p^{2}, d t=2 w d w$ and $d \theta=2 p d p$ in (3.4.3)

$$
=4 \int_{0}^{\infty} \int_{0}^{\infty} e^{-\left(w^{2}+p^{2}\right)} w^{2 s-1} p^{2 q-1} d s d p
$$

Using the plane polar coordinates $r, \theta$ given by $w=r \cos \theta, p=r \sin \theta$ and $d w d p=$ $r d r d \theta$. It becames

$$
\begin{aligned}
\Gamma(s) \Gamma(q) & =4 \int_{0}^{\infty} \int_{0}^{\frac{\pi}{2}} e^{-r^{2}}(r \cos \theta)^{2 s-1}(r \sin \theta)^{2 q-1} r d r d \theta \\
& =4 \int_{0}^{\infty} \int_{0}^{\frac{\pi}{2}} e^{-r^{2}} r^{2 s+2 q-1}(\cos \theta)^{2 s-1}(\sin \theta)^{2 q-1} d r d \theta \\
& =2 \int_{0}^{\infty} e^{-r^{2}} r^{2 s+2 q-1} d r 2 \int_{0}^{\frac{\pi}{2}}(\cos \theta)^{2 s-1}(\sin \theta)^{2 q-1} d \theta
\end{aligned}
$$


Putting $t=r^{2}$ and $d t=2 r d r$ in the first integral,

$$
\begin{aligned}
& =2 \int_{0}^{\infty} e^{-(\sqrt{t})^{2}} t^{\frac{1}{2}(2 s+2 q-1)} \frac{d t}{2 \sqrt{t}} 2 \int_{0}^{\frac{\pi}{2}}(\cos \theta)^{2 s-1}(\sin \theta)^{2 q-1} d \theta \\
& =2 \int_{0}^{\infty} e^{-t} t^{s+q-\frac{1}{2}} t^{\frac{-1}{2}} \frac{d t}{2} 2 \int_{0}^{\frac{\pi}{2}}(\cos \theta)^{2 s-1}(\sin \theta)^{2 q-1} d \theta \\
& =\int_{0}^{\infty} e^{-t} t^{s+q-1} d t 2 \int_{0}^{\frac{\pi}{2}}(\cos \theta)^{2 s-1}(\sin \theta)^{2 q-1} d \theta
\end{aligned}
$$

On the other hand second integral gives $B(s, q)$. So,

$$
\Gamma(s) \Gamma(q)=\Gamma(s+q) B(s, q)
$$

Hence

$$
\frac{\Gamma(s) \Gamma(q)}{\Gamma(s+q)}=B(s, q)
$$

gives the relation between beta and gamma function.

\subsection{The Infinite Product Expression of Beta Function}

Theorem 3.5.1 [1]For $s, q \notin \mathbb{Z}_{0}=\{0,-1,-2, \ldots\}$,

$$
B(s, q)=\frac{s+q}{s q} \prod_{n=1}^{\infty} \frac{\left(1+\frac{s+q}{n}\right)}{\left(1+\frac{s}{n}\right)\left(1+\frac{q}{n}\right)} .
$$


Proof. Firstly, putting Euler infinitive product expression of $\Gamma(s)$ in to formula (3.4.2) then, we get

$$
\frac{\Gamma(s) \Gamma(q)}{\Gamma(s+q)}=\frac{\frac{1}{s} \frac{1}{q}}{\frac{1}{s+q}} \frac{\prod_{n=1}^{\infty}\left[\left(1+\frac{1}{n}\right)^{s}\left(1+\frac{1}{n}\right)^{q}\left(1+\frac{s}{n}\right)^{-1}\left(1+\frac{q}{n}\right)^{-1}\right]}{\prod_{n=1}^{\infty}\left[\left(1+\frac{1}{n}\right)^{s+q}\left(1+\frac{s+q}{n}\right)^{-1}\right]} .
$$

Hence

$$
B(s, q)=\frac{s+q}{s q} \prod_{n=1}^{\infty} \frac{\left(1+\frac{s+q}{n}\right)}{\left(1+\frac{s}{n}\right)\left(1+\frac{q}{n}\right)}
$$

The next two theorems give the reccurence relation for beta function (3.4.2).

Theorem 3.5.2 [1] For $\operatorname{Re}(s)>0$ and $\operatorname{Re}(q)>0$,

$$
B(s+1, q)=\frac{s}{s+q} B(s, q)=\frac{s}{q} B(s, q+1)
$$

Proof. Using Theorem 3.4.2, we get

$$
\begin{aligned}
B(s+1, q) & =\frac{\Gamma(s+1) \Gamma(q)}{\Gamma(s+q+1)} \\
& =\frac{s \Gamma(s) \Gamma(q)}{s+q \Gamma(s+q)} \\
& =\frac{s}{s+q} B(s, q)
\end{aligned}
$$


and

$$
\begin{aligned}
\frac{s}{s+q} B(s, q) & =\frac{s}{s+q} \frac{\Gamma(s) \Gamma(q)}{\Gamma(s+q)} \\
& =\frac{\Gamma(s+1) \Gamma(q)}{\Gamma(s+(q+1))} \\
& =\frac{s}{q} \frac{\Gamma(s) \Gamma(q+1)}{\Gamma(s+(q+1))} \\
& =\frac{s}{q} B(s, q+1)
\end{aligned}
$$

which is exactly same in Theorem 3.5.2

Theorem 3.5.3 [1] For $\operatorname{Re}(s)>0$ and $\operatorname{Re}(q)>0$,

$$
B(s+1, q)+B(s, q+1)=B(s, q)
$$

Proof. Using Theorem 3.4.2, we find

$$
\begin{aligned}
B(s+1, q)+B(s, q+1) & =\frac{\Gamma(s+1) \Gamma(q)}{\Gamma(s+q+1)}+\frac{\Gamma(s) \Gamma(q+1)}{\Gamma(s+q+1)} \\
& =\frac{\Gamma(s+1) \Gamma(q)+\Gamma(s) \Gamma(q+1)}{\Gamma(s+q+1)} \\
& =\frac{s \Gamma(s) \Gamma(q)+\Gamma(s) q \Gamma(q+1)}{(s+\beta) \Gamma(s+q)} \\
& =\frac{\Gamma(s) \Gamma(\beta)(s+q)}{(s+\beta) \Gamma(s+q)} \\
& =\frac{\Gamma(s) \Gamma(q)}{\Gamma(s+q)}
\end{aligned}
$$

Hence

$$
B(s+1, q)+B(s, q+1)=B(s, q)
$$

which is exactly same in Theorem 3.5.2 


\section{Chapter 4}

\section{GENERALIZED GAMMA FUNCTION}

In this chapter, we give definition and properties the generalized gamma function.

\subsection{Definition of the Generalized Gamma Function}

Definition 4.1.1 [1]The generalized gamma function can be defined by

$$
\Gamma_{c}(s)=\int_{0}^{\infty} t^{s-1} e^{-t-\frac{c}{t}} d t \quad(\operatorname{Re}(c)>0 ; c=0, \operatorname{Re}(s)>0) .
$$

Notice that in the case $c=0$ the function conclude with the classical gamma function.

Definition 4.1.2 [1] For $\operatorname{Re}(c)>0$ and $|\arg (\sqrt{c})|<\pi$,

$$
\Gamma_{c}(s)=2 c^{s / 2} K_{s}(2 \sqrt{c}) .
$$

where $K_{s}$ (Theorem 2.0.5) [5] is a Macdonald function.

Theorem 4.1.3 If $\operatorname{Re}(c)>0$ or $c=0$ and $\operatorname{Re}(s)>0$ then, we have

$$
\Gamma_{a c}(s)=a^{s} \int_{0}^{\infty} t^{s-1} e^{-a t-\frac{c}{t}} d t
$$

Proof. The substitution $t=x^{2}$ and $d t=2 x d x$ in (4.1.1), we obtain another expression

$$
\Gamma_{c}(s)=2 \int_{0}^{\infty} x^{2 s-1} e^{-x^{2}-c x^{-2}} d x
$$


The substitution $t / \sqrt{c}=u$ and $d t=\sqrt{c} d u$ in (4.1.1) gives that

$$
\begin{aligned}
\Gamma_{c}(s) & =\int_{0}^{\infty} u^{s-1} c^{\frac{s}{2}-\frac{1}{2}} e^{-\sqrt{c} u-\frac{1}{\sqrt{c} u}} d u \\
& =c^{\frac{s}{2}} \int_{0}^{\infty} u^{s-1} e^{-\sqrt{c}\left(u+u^{-1}\right)} d u \quad(\operatorname{Re}(c)>0)
\end{aligned}
$$

After putting $u=e^{y}$ and $d u=e^{y} d y$ in (4.1.4), we obtain following;

$$
\begin{gathered}
\Gamma_{c}(s)=c^{\frac{\alpha}{2}} \int_{-\infty}^{\infty} e^{x(s-1)} e^{-\sqrt{c}\left(e^{y}+e^{-y}\right)} e^{x} d y \\
=c^{\frac{s}{2}} \int_{-\infty}^{\infty} e^{y s} e^{-\sqrt{c}\left(e^{y}+e^{-y}\right)} d y \\
\Gamma_{c}(s)=c^{\frac{s}{2}} \int_{-\infty}^{\infty} e^{(y s-2 \sqrt{c} \cosh y)} d y \quad(\operatorname{Re}(c)>0)
\end{gathered}
$$

where $\cosh y=\frac{\left(e^{y}+e^{-y}\right)}{2}$.

For $|a|+|c| \neq 0$ or $c=0, \operatorname{Re}(a)>0, \operatorname{Re}(s)>0$, then

$$
\Gamma_{a c}(s)=a^{s} \int_{0}^{\infty} t^{s-1} e^{-a t-\frac{c}{t}} d t
$$

which is the desired result.

\subsection{Properties of the Generalized Gamma Function}

In this section, we studied some reccurrence relations of the generalized Gamma function.

Theorem 4.2.1 [1] (The diffirence formula)

$$
\Gamma_{c}(s+1)=s \Gamma_{c}(s)+c \Gamma_{c}(s-1)
$$


Proof. If we choose $f(x)=e^{-x-c x^{-1}}$ in Mellin transform (2.0.4) operator, we find

$$
\begin{aligned}
M\left\{e^{-x-c x^{-1}}, s\right\} & =\left\langle x_{+}^{s-1}, e^{-x-c x^{-1}}\right\rangle \\
& =\int_{0}^{\infty} x^{s-1} f(x) d x=\Gamma_{c}(s) \\
& =M\{f(x) ; s\}
\end{aligned}
$$

and

$$
\begin{aligned}
M\left\{f^{\prime}(x) ; s\right\} & =\int_{0}^{\infty} x^{s-1} f(x) d x=-(s-1) \Gamma_{c}(s-1) \\
& =-(s-1) M\{f(x) ; s-1\}
\end{aligned}
$$

In above the integrals, if we apply integration by parts $\left(u=x^{s-1}\right.$ and $\left.d v=f^{\prime}(x)\right)$, we find

$$
\begin{aligned}
\int_{0}^{\infty} x^{s-1} f(x) d x & =\left.x^{s-1} f(x)\right|_{0} ^{\infty}-\int_{0}^{\infty} f(x)(s-1) x^{s-2} d t \\
& =-(s-1) \int_{0}^{\infty} x^{s-2} e^{-x-c x^{-1}} d t \\
& =-(s-1) \Gamma_{c}(s-1) \\
& =M\left\{\left(-1+c x^{-2}\right) e^{-x-c x^{-1}} ; s\right\}
\end{aligned}
$$

which simplifies to give

$$
\begin{gathered}
-(s-1) \Gamma_{c}(s-1)=-\Gamma_{c}(s)+c \Gamma_{c}(s-2) \\
\Gamma_{c}(s)=(s-1) \Gamma_{c}(s-1)+c \Gamma_{c}(s-2) .
\end{gathered}
$$


Finally, replacing $s=s+1$ in (4.2.3), we get the proof of (4.2.1).

Theorem 4.2.2 [1] (Log-convex property) Let $1<n<\infty$ and $\left(\frac{1}{n}\right)+\left(\frac{1}{m}\right)=1$, then

$$
\Gamma_{c}\left(\frac{\alpha}{n}+\frac{\beta}{m}\right) \leqslant\left(\Gamma_{c}(\alpha)\right)^{\frac{1}{n}}\left(\Gamma_{c}(\beta)\right)^{\frac{1}{m}} \quad(c \geqslant 0, \alpha>0, \beta>0)
$$

Proof. For $s=\left(\frac{\alpha}{p}+\frac{\beta}{q}\right)$,

$$
\begin{aligned}
\Gamma_{c}\left(\frac{\alpha}{n}+\frac{\beta}{m}\right) & =\int_{0}^{\infty} t^{\frac{\alpha}{n}+\frac{\beta}{m}-1} e^{-t-\frac{c}{t}} d t \\
& =\int_{0}^{\infty}\left(t^{\alpha-1} e^{-t-\frac{c}{t}}\right)^{\frac{1}{n}}\left(t^{\beta-1} e^{-t-\frac{c}{t}}\right)^{\frac{1}{m}} d t
\end{aligned}
$$

Using Hölder inequality (2.0.6)

$$
\Gamma_{c}\left(\frac{\alpha}{n}+\frac{\beta}{m}\right) \leqslant(\underbrace{\int_{0}^{\infty} t^{\alpha-1} e^{-t-\frac{c}{t}}}_{\Gamma_{c}(\alpha)})^{\frac{1}{n}}(\underbrace{\int_{0}^{\infty} t^{\beta-1} e^{-t-\frac{c}{t}}}_{\Gamma_{c}(\beta)})^{\frac{1}{m}} d t
$$

which is exactly same in (4.2.4).

Remark 4.2.3 [1] The inequality (4.2.4), has several useful special cases. For example, setting $n=m=2$,we find

$$
\Gamma_{c}\left(\frac{\alpha+\beta}{2}\right) \leqslant \sqrt{\Gamma_{c}(\alpha) \Gamma_{c}(\beta)},(\alpha>0, \beta>0, c \geqslant 0)
$$

But, as the geometric mean of two positive numbers is less than or equal to their aritmetic mean, we find

$$
\Gamma_{c}\left(\frac{\alpha+\beta}{2}\right) \leqslant\left(\Gamma_{c}(\alpha) \Gamma_{c}(\beta)\right)^{\frac{1}{2}} \leqslant \frac{\left(\Gamma_{c}(\alpha)+\Gamma_{c}(\beta)\right)}{2}, \quad(\alpha>0, \beta>0, c \geqslant 0)
$$


Corollary 4.2.4 [1]For $1<n<\infty$ and $\left(\frac{1}{n}\right)+\left(\frac{1}{m}\right)=1$,

$$
K_{\frac{x}{n}+\frac{y}{m}}(t) \leqslant\left(K_{x}(t)\right)^{\frac{1}{n}}\left(K_{y}(t)\right)^{\frac{1}{m}} \quad(x>0, y>0, t>0) .
$$

Proof. In this proof, its necessary the use the inequality(4.2.4) and Macdonald Function representation of Generalized Gamma Functions (4.1.2) as the following.

$$
\Gamma_{c}(s)=2 c^{\frac{s}{2}} K_{s}(2 \sqrt{c})
$$

and

$$
\Gamma_{c}\left(\frac{x}{n}+\frac{y}{m}\right) \leqslant\left[\Gamma_{c}(x)\right]^{\frac{1}{n}}\left[\Gamma_{c}(y)\right]^{\frac{1}{m}}
$$

Substitution $s=\frac{x}{n}+\frac{y}{m}$ and $t=2 \sqrt{c}$ in Macdonald Function representation of Gamma Functions, we find

$$
\Gamma_{c}\left(\frac{x}{n}+\frac{y}{m}\right)=2 c^{\frac{1}{2}\left(\frac{x}{n}+\frac{y}{m}\right)} K_{\frac{x}{n}+\frac{y}{m}}(2 \sqrt{c}) .
$$

Using the log-convex property (4.2.4) then

$$
\Gamma_{c}\left(\frac{x}{n}+\frac{y}{m}\right)=2 c^{\frac{1}{2}\left(\frac{x}{n}+\frac{y}{m}\right)} K_{\frac{x}{n}+\frac{y}{m}}(2 \sqrt{c}) \leqslant\left[\Gamma_{c}(x)\right]^{\frac{1}{n}}\left[\Gamma_{c}(y)\right]^{\frac{1}{m}}
$$

Take $x=2 \sqrt{c}$ and $y=2 \sqrt{c}$ in the Macdonald function representation in $\Gamma_{c}(x)$ and $\Gamma_{c}(y)$, then

$$
2 c^{\frac{1}{2}\left(\frac{x}{n}+\frac{y}{m}\right)} K_{\frac{x}{n}+\frac{y}{m}}(2 \sqrt{c}) \leqslant\left[2 c^{\frac{x}{2}} K_{x}(2 \sqrt{c})\right]^{\frac{1}{n}}\left[2 c^{\frac{y}{2}} K_{y}(2 \sqrt{c})\right]^{\frac{1}{m}}
$$


Hence,

$$
K_{\frac{x}{n}+\frac{y}{m}}(2 \sqrt{c}) \leqslant\left[K_{x}(2 \sqrt{c})\right]^{\frac{1}{n}}\left[K_{y}(2 \sqrt{c})\right]^{\frac{1}{m}}
$$

gives the result.

Corollary 4.2.5 [1] For $x, y, t>0$

$$
K_{\frac{1}{2}(x+y)}(t) \leqslant \sqrt{K_{x}(t) K_{y}(t)}
$$

Proof. The substitution $n=m=2$ in (4.2.5), we obtain

$$
K_{\frac{x}{2}+\frac{y}{2}}(t) \leqslant\left[K_{x}(t)\right]^{\frac{1}{2}}\left[K_{y}(t)\right]^{\frac{1}{2}}=\sqrt{K_{x}(t) K_{y}(t)} .
$$

Theorem 4.2.6 [1] (The reflection formula) For $\operatorname{Re}(c)>0$,

$$
c^{s} \Gamma_{c}(-s)=\Gamma_{c}(s) .
$$

Proof. The substitutions $t=\frac{c}{u}$ and $d t=\frac{-c}{u^{2}} d u$ in $\Gamma_{c}(s)$, we find

$$
\begin{aligned}
& \Gamma_{c}(s)=\int_{0}^{\infty} \frac{c^{s-1}}{u^{s-1}} e^{\frac{-c}{u}-u} \frac{c}{u^{2}} d u \\
& \Gamma_{c}(s)=\int_{0}^{\infty} \frac{c^{s}}{u^{s+1}} e^{\frac{-c}{u}-u} d u \\
& \Gamma_{c}(s)=c^{s} \int_{0}^{\infty} u^{-s-1} e^{\frac{-c}{u}-u} d u
\end{aligned}
$$


Hence

$$
\Gamma_{c}(s)=c^{s} \Gamma_{c}(-s)
$$

gives the result.

Corollary 4.2.7 [1] For $\operatorname{Re}(b)>0$,

$$
\Gamma_{c}(1-s)=c^{-s}\left[\Gamma_{c}(s+1)-s \Gamma_{c}(s)\right]
$$

Proof. Replacing $s$ by $-s$ in the difference formula (4.2.1) and $s$ by $s+1$ the reflection formula (4.2.10), we get

$$
c^{-s} \Gamma_{c}(s)=\Gamma_{c}(-s)
$$

and

$$
\Gamma_{c}(1-s)=-s \Gamma_{c}(-s)+c \Gamma_{c}(-s-1)
$$

so

$$
\Gamma_{c}(1-s)=-s\left[c^{-s} \Gamma_{c}(-s)\right]+c \Gamma_{c}(-s-1) .
$$

According to (4.2.12), we find

$$
\begin{aligned}
\Gamma_{c}(1-s) & =-s\left[c^{-s} \Gamma_{c}(s)\right]+c^{-(s+1)} \Gamma_{c}(s+1) \\
& =-s c^{-s} \Gamma_{c}(s)+c^{-s} \Gamma_{c}(s+1) .
\end{aligned}
$$


Hence

$$
\Gamma_{c}(1-s)=c^{-s}\left[-s \Gamma_{c}(s)+\Gamma_{c}(s+1)\right]
$$

Theorem 4.2.8 [1] (Product Formula) For $c>0 ; c=0, \operatorname{Re}(s)>0$ and $\operatorname{Re}(q)>0$,

$$
\Gamma_{c}(s) \Gamma_{c}(q)=2 \int_{0}^{\infty} \tau^{2(s+q)} e^{-\tau^{2} B} B\left(s, q ; \frac{c}{\tau^{2}}\right) d \tau
$$

where

$$
B(x, y ; c)=\int_{0}^{1} t^{x-1}(1-t)^{y-1} e^{-\frac{c}{t(1-t)}} d t
$$

is the Extended Beta Function.

Proof. According to definition of Generalized Gamma Function, we find

$$
\Gamma_{c}(s) \Gamma_{c}(q)=\int_{0}^{\infty} t^{s-1} e^{-t-\frac{c}{t}} d t \int_{0}^{\infty} t^{q-1} e^{-v-\frac{c}{v}} d v
$$

The transformation $t=k^{2}$ and $v=\ell^{2}$ in (4.2.15) yields

$$
\begin{aligned}
\Gamma_{c}(s) \Gamma_{c}(q) & =\int_{0}^{\infty}\left(k^{2}\right)^{s-1} \exp \left[-k^{2}-\frac{c}{k^{2}}\right] 2 k d k \int_{0}^{\infty}\left(\ell^{2}\right)^{q-1} \exp \left[-\ell^{2}-\frac{c}{\ell^{2}}\right] 2 \ell d \ell \\
& =4 \int_{0}^{\infty} \int_{0}^{\infty} k^{2^{s-1}} \ell^{2 q-1} e^{-\left(k^{2}+\ell^{2}\right)} e^{-c\left(\frac{1}{k^{2}}+\frac{1}{\ell^{2}}\right)} d k d \ell .
\end{aligned}
$$


The substitution $k=\tau \cos \theta, \ell=\tau \sin \theta$ and $d k d \ell=\tau d \tau d \theta$, we find

$$
\begin{aligned}
& =4 \int_{0}^{\infty} \int_{0}^{\frac{\pi}{2}}(\tau \cos \theta)^{2 s-1}(\tau \sin \theta)^{2 q-1} e^{\left[-r^{2} \cos ^{2} \theta-\tau^{2} \sin ^{2} \theta\right]} e^{\left[-\frac{c}{\tau^{2} \cos \theta}-\frac{c}{\tau^{2} \sin \theta}\right]} \tau d \tau d \theta \\
& =4 \int_{0}^{\infty} \int_{0}^{\frac{\pi}{2}} \tau^{2(s+q)-1}(\cos \theta)^{2 s-1}(\sin \theta)^{2 q-1} e^{\left[-\tau^{2}\left(\cos ^{2} \theta+\sin ^{2} \theta\right)\right]} e^{\left[-\frac{c}{\tau^{2} \cos ^{2} \theta}-\frac{c}{\tau^{2} \sin ^{2} \theta}\right]} d \tau d \theta \\
& =2 \int_{0}^{\infty} \tau^{2(s+q)-1} e^{-\tau^{2}} d \tau\left\{2 \int_{0}^{\frac{\pi}{2}}(\cos \theta)^{2 s-1}(\sin \theta)^{2 q-1} e^{\left[\left(-c\left(\frac{\tau^{2}\left(\sin ^{2} \theta+\cos ^{2} \theta\right)}{\tau^{4} \cos ^{2} \theta \sin ^{2} \theta}\right)\right)\right]} d \theta\right\} \\
& =2 \int_{0}^{\infty} \tau^{2(s+q)-1} e^{-\tau^{2}} d \tau\left\{2 \int_{0}^{\frac{\pi}{2}}(\cos \theta)^{2 s-1}(\sin \theta)^{2 q-1} e^{\left[\left(-\frac{c}{\tau^{2}} \frac{1}{\cos ^{2} \theta \sin ^{2} \theta}\right)\right]} d \theta\right\} \\
& =2 \int_{0}^{\infty} \tau^{2(s+q)-1} e^{-\tau^{2}} d \tau\left\{2 \int_{0}^{\frac{\pi}{2}}(\cos \theta)^{2 s-1}(\sin \theta)^{2 q-1} e^{\left[-\frac{c}{\tau^{2}} \frac{1}{\left(\frac{1}{2} \sin 2 \theta\right)^{2}}\right]} d \theta\right\} \\
& =2 \int_{0}^{\infty} \tau^{2(s+q)-1} e^{-\tau^{2}} d \tau\left\{2 \int_{0}^{\frac{\pi}{2}}(\cos \theta)^{2 s-1}(\sin \theta)^{2 q-1} e^{\left[\left(-\frac{c}{\tau^{2}} \frac{4}{\sin 2 \theta}\right)\right]} d \theta\right\} \\
& =2 \int_{0}^{\infty} \tau^{2(s+q)-1} e^{-\tau^{2}} d \tau\left\{2 \int_{0}^{\frac{\pi}{2}}(\cos \theta)^{2 s-1}(\sin \theta)^{2 q-1} e^{\left(-\frac{4 c}{\tau^{2}} \csc 2 \theta\right)} d \theta\right\}
\end{aligned}
$$

But, the inner integral in the above equation gives $B\left(s, q ; \frac{c}{\tau^{2}}\right)$. Hence

$$
\Gamma_{c}(s) \Gamma_{c}(q)=2 \int_{0}^{\infty} \tau^{2(s+q)-1} \exp \left(-\tau^{2}\right) B\left(s, q ; \frac{c}{\tau^{2}}\right) d \tau
$$

Theorem 4.2.9 For $\operatorname{Re}(b)>0$,

$$
\Gamma_{c}^{2}(s)=4 c^{s} \int_{0}^{\infty} e^{-\left(\tau^{2}+\frac{2 c}{\tau^{2}}\right)} K_{s}\left(\frac{2 c}{\tau^{2}}\right) \frac{d \tau}{\tau}
$$

Proof. The substitution $q=-s$ in (4.2.14) yields

$$
\Gamma_{c}(s) \Gamma_{c}(-s)=2 \int_{0}^{\infty} \tau^{2(s-s)-1} e^{-\tau^{2}} B\left(s,-s ; \frac{c}{\tau^{2}}\right) d \tau
$$


But, the Extended Beta Function for $q=-s$ is expressible in terms of the Macdonald function to give [15]. In [15], M. Chaudhry and S. Zubair gives the Macdonald function expression of $B\left(s,-s ; \frac{c}{\tau^{2}}\right)$

$$
B\left(s,-s ; \frac{c}{\tau^{2}}\right)=2 e^{\left(-\frac{2 c}{\tau^{2}}\right)} K_{s}\left(\frac{2 c}{\tau^{2}}\right)(\operatorname{Re}(c>0))
$$

so

$$
\Gamma_{c}(s) \Gamma_{c}(-s)=2 \int_{0}^{\infty} e^{-\tau^{2}} \tau^{-1} 2 \exp \left(-\frac{2 c}{\tau^{2}}\right) K_{s}\left(\frac{2 c}{\tau^{2}}\right) d \tau
$$

According to reflection formula (4.2.10), we get

$$
\begin{gathered}
\Gamma_{c}(s) c^{-s} \Gamma_{c}(s)=4 \int_{0}^{\infty} e^{-\tau^{2}} \exp \left(-\frac{2 c}{\tau^{2}}\right) K_{s}\left(\frac{2 c}{\tau^{2}}\right) \frac{d \tau}{\tau} \\
\Gamma_{c}^{2}(s) c^{-s}=4 \int_{0}^{\infty} \exp \left(-\tau^{2}-\frac{2 c}{\tau^{2}}\right) K_{s}\left(\frac{2 c}{\tau^{2}}\right) \frac{d \tau}{\tau} .
\end{gathered}
$$

Hence

$$
\Gamma_{c}^{2}(s)=4 c^{\alpha} \int_{0}^{\infty}\left(-\tau^{2}-\frac{2 c}{\tau^{2}}\right) K_{s}\left(\frac{2 c}{\tau^{2}}\right) \frac{d \tau}{\tau}
$$

Theorem 4.2.10 [1] For $\operatorname{Re}(c)>0$,

$$
\Gamma_{c}^{2}(s)=\pi^{\frac{1}{2}} 2^{1-s} c^{\frac{1}{2}(s-1)} \int_{0}^{\infty} \exp \left[-\left(\tau^{2}+\frac{2 c}{\tau^{2}}\right)\right] \times W_{-\frac{s}{2}, \frac{s}{2}}\left(\frac{4 c}{\tau^{2}}\right) d \tau
$$

where $W_{\lambda, \mu}$ is one of the Whittaker Functions [5] 
Proof. Putting $q=s$ in (4.2.14) we find

$$
\begin{gathered}
\Gamma_{c}(s) \Gamma_{c}(s)=2 \int_{0}^{\infty} \tau^{2(s+s)-1} \exp \left(-\tau^{2}\right) B\left(s, s ; \frac{c}{\tau^{2}}\right) d \tau \\
\Gamma_{c}^{2}(s)=2 \int_{0}^{\infty} \tau^{4 s-1} \exp \left(-\tau^{2}\right) B\left(s, s ; \frac{c}{\tau^{2}}\right) d \tau
\end{gathered}
$$

On the other hand, Ryzhink's and Gradshteyn ([5]) was obtained the following results,

$$
B\left(s, s ; \frac{c}{\tau^{2}}\right)=\pi^{\frac{1}{2}} 2^{-s} c^{\frac{1}{2}(s-1)} e^{-\frac{2 c}{\tau^{2}} W_{-\frac{s}{2}, \frac{s}{2}}}\left(\frac{4 c}{\tau^{2}}\right)
$$

Hence,

$$
\Gamma_{c}^{2}(\alpha)=2^{1-s} \pi^{\frac{1}{2}} c^{\frac{1}{2}(s-1)} \int_{0}^{\infty} \exp \left[-\tau^{2}-2 c\right] \times W_{-\frac{s}{2}, \frac{s}{2}}(4 c) d \tau
$$

\subsection{Mellin and Laplace Transforms}

Theorem 4.3.1 [16] (Mellin transform representation) For $\operatorname{Re}(\alpha)>0$ and $\operatorname{Re}(s+\alpha)>$ 0 ,

$$
M\left\{\Gamma_{c}(s) ; \alpha\right\}=\Gamma(\alpha) \Gamma(s+\alpha) \quad(\operatorname{Re}(\alpha)>0, \operatorname{Re}(s+\alpha)>0)
$$

Proof. According to the definition of the Mellin transform of $\Gamma_{c}(\alpha)(4.1 .1)$, we get

$$
M\left\{\Gamma_{c}(s) ; \alpha\right\}=\left\langle c_{+}^{\alpha-1}, \Gamma_{c}(s)\right\rangle .
$$


Using the formula (4.2.2), we find

$$
M\left\{\Gamma_{c}(s) ; \alpha\right\}=\left\langle c_{+}^{\alpha-1}, \Gamma_{c}(s)\right\rangle=\left\langle c_{+}^{\alpha-1},\left\langle t_{+}^{s-1}, e^{-t-\frac{c}{t}}\right\rangle\right\rangle .
$$

Applying of the Fubini's Theorem [4] we find

$$
M\left\{\Gamma_{c}(s) ; \alpha\right\}=\left\langle t_{+}^{s-1}, e^{-t}\left\langle c_{+}^{\alpha-1}, e^{-\frac{c}{t}}\right\rangle\right\rangle
$$

But, according to (3.1.2)

$$
\left\langle c_{+}^{\alpha-1}, e^{-\frac{c}{t}}\right\rangle=\int_{0}^{\infty} c_{+}^{\alpha-1} e^{-\frac{c}{t}} d c
$$

The substitution $u=\frac{c}{t}$ and $d u=\frac{1}{t} d t$ in (4.3.3) yields

$$
\left\langle c_{+}^{\alpha-1}, e^{-\frac{c}{t}}\right\rangle=\int_{0}^{\infty} t^{\alpha} u^{\alpha-1} e^{-u} d u=t^{\alpha} \Gamma(\alpha)
$$

Hence, for $\operatorname{Re}(\alpha)>0$ and $\operatorname{Re}(s+\alpha)>0$,

$$
M\left\{\Gamma_{c}(s) ; \alpha\right\}=\Gamma(\alpha)\left\langle t_{+}^{s+\alpha-1}, e^{-t}\right\rangle=\Gamma(\alpha) \Gamma(s+\alpha)
$$

Corollary 4.3.2 [1] For $\operatorname{Re}(s)>-1$,

$$
\int_{0}^{\infty} \Gamma_{c}(s) d c=\Gamma(s+1)
$$


Proof. Setting $\alpha=1$ in $(4.3 .1)$, we find

$$
M\left\{\Gamma_{c}(s) ; 1\right\}=\int_{0}^{\infty} c_{+}^{1-1} \Gamma_{c}(s) d c=\Gamma(1) \Gamma(s+1)
$$

Hence,

$$
\int_{0}^{\infty} \Gamma_{c}(s) d c=\Gamma(s+1)
$$

Theorem 4.3.3 [10] (Laplace transform representation) Let $L$ be the Laplace transform operator, for $\operatorname{Re}(y)>0, \operatorname{Re}(y+s)>0$ and $\operatorname{Re}(p)>0$,

$$
L\left\{t^{y-1} \Gamma_{t}(s) ; p\right\}=\Gamma(y) \Gamma(y+s) p^{-\frac{1}{2}(2 y+s-1)} e^{\frac{1}{2 p}} W_{-\frac{1}{2}(2 y+s-1), \frac{s}{2}}\left(\frac{1}{p}\right)
$$

Proof. Using the definition of the Laplace transform (2.0.1) and Macdonald representation of $\Gamma_{t}(s)$ in (4.1.2), we find

$$
\begin{aligned}
L\left\{t^{y-1} \Gamma_{t}(s) ; p\right\} & =\int_{0}^{\infty} e^{-p t} t^{y-1} \Gamma_{t}(s) d t \\
& =2 \int_{0}^{\infty} t^{y+\frac{s}{2}-1} e^{-p t} K_{s}(2 \sqrt{t})
\end{aligned}
$$

The The integral in (4.3.6) is a special case of $([5], p .741(6.643))$ when we take $\mu-$ $\frac{1}{2}=y+\frac{s}{2}-1, y=\frac{s}{2}, s=p, \beta=1$.So

$$
L\left\{t^{y-1} \Gamma_{t}(s) ; p\right\}=\Gamma\left(\frac{s}{2}+\frac{1}{2}+y+\frac{s}{2}-\frac{1}{2}\right) \Gamma\left(\frac{s}{2}\right) e^{-\frac{1}{2 p}} p^{-y-\frac{s}{2}+\frac{1}{2}} W_{y+\frac{s}{2}-\frac{1}{2}, \frac{s}{2}}\left(\frac{1}{p}\right)
$$


Hence,

$$
L\left\{t^{y-1} \Gamma_{t}(s) ; p\right\}=\Gamma(y) \Gamma(y+s) p^{-\frac{1}{2}(2 y+s-1)} e^{\frac{1}{2 p}} W_{-\frac{1}{2}(2 y+s-1), \frac{s}{2}}\left(\frac{1}{p}\right) .
$$




\section{Chapter 5}

\section{THE DIGAMMA FUNCTION}

\subsection{Definition of the Digamma Function}

First derivative of $\ln \Gamma(s)$ is called Digamma Function and is denoted by $\psi(s)$.

$$
\psi(s)=\frac{d}{d s}\{\ln \Gamma(s)\}=\frac{\Gamma^{\prime}(s)}{\Gamma(s)}
$$

\subsection{Properties of the Digamma Function}

Definition 5.2.1 [3] For $s \in \mathbb{C}$,

$$
\psi(s)=-\gamma-\frac{1}{s}+\sum_{n=1}^{\infty}\left(\frac{1}{n}-\frac{1}{n+s}\right)=\lim _{n \rightarrow \infty}\left(\log n-\sum_{k=0}^{n} \frac{1}{k+s}\right)
$$

where $\gamma=0,5772156 \ldots$ and $s \neq 0,-1,-2,-3, \ldots$

Proof. Take the logarithm of Weierstrass expression $([5]$, p.97) of $\Gamma(s)$, we find

$$
\log \Gamma(s)=\log s^{-1}+\log e^{-\gamma s}+\sum_{n=1}^{\infty} \log \frac{n}{n+s}+\sum_{n=1}^{\infty} \log e^{\frac{s}{n}}
$$

By differentiating the series (5.2.2), we find

$$
\begin{gathered}
\psi(s)=\frac{\Gamma^{\prime}(s)}{\Gamma(s)}=\frac{-s^{-2}}{s^{-1}} \frac{-\gamma e^{-\gamma s}}{e^{-\gamma s}}+\sum_{n=1}^{\infty} \frac{n}{(n+s)^{2}}\left(\frac{n+s}{-n}\right)+\sum_{n=1}^{\infty} \frac{1 / n e^{s / n}}{e^{s / n}} \\
\psi(s)=-\gamma-\frac{1}{s}+\sum_{n=1}^{\infty}\left(\frac{1}{n}-\frac{1}{n+s}\right) .
\end{gathered}
$$


Thus, proof of the first part is completed. For the second part, substitution limit expression of $\gamma([5])$ in $(5.2 .1)$, then

$$
\psi(s)=-\lim _{n \rightarrow \infty}\left(\sum_{k=1}^{n} \frac{1}{k}-\log n\right)+\sum_{n=1}^{\infty} \frac{1}{n}-\sum_{n=0}^{\infty} \frac{1}{n+s}
$$

Hence

$$
\psi(s)=\lim _{n \rightarrow \infty}\left(\log n-\sum_{k=0}^{n} \frac{1}{k+s}\right) .
$$

Next, we give representation of $\psi(s)$ for the series.

Theorem 5.2.2 [1] For $s \in \mathbb{C}$ away from $s=0,-1,-2, \ldots$

$$
\psi(s)=-\gamma+(s-1) \sum_{n=o}^{\infty} \frac{1}{(n+1)(s+n)}
$$

Proof. Using the first part of theorem 5.2.1, we find

$$
\begin{aligned}
\psi(s) & =-\gamma-\frac{1}{s}+\sum_{n=1}^{\infty} \frac{1}{s}-\sum_{n=1}^{\infty} \frac{1}{s+n} \\
& =-\gamma+\sum_{n=1}^{\infty} \frac{1}{n}-\sum_{n=0}^{\infty} \frac{1}{s+n} \\
& =-\gamma+\sum_{n=0}^{\infty} \frac{1}{n+1}-\sum_{n=0}^{\infty} \frac{1}{s+n} \\
& =-\gamma+\sum_{n=o}^{\infty} \frac{(s-1)}{(n+1)(s+n)}
\end{aligned}
$$

Hence

$$
\psi(s)=-\gamma+(s-1) \sum_{n=o}^{\infty} \frac{1}{(n+1)(s+n)} .
$$


The following theorems gives integral representation of Digamma Function .

Proposition 5.2.3 [12] Let $p, q \in \mathbb{R}$, then

$$
\int_{0}^{1} \frac{t^{p-1}-t^{q-1}}{1-t} d t=\psi(q)-\psi(p)
$$

Theorem 5.2.4 [12] For $\operatorname{Re} s>-1$

$$
\psi(s+1)=-\gamma+\int_{0}^{1} \frac{1-t^{\alpha}}{1-t} d t
$$

Proof. For $p=1$ in Proposition 5.2.4, we find

$$
\int_{0}^{1} \frac{t^{0}-t^{q-1}}{1-t} d t=\psi(q)-\psi(1)
$$

Using the particular value of $\psi(1)=-\gamma$

$$
\int_{0}^{1} \frac{t-t^{q-1}}{1-t} d t=\psi(q)+\gamma
$$

Lets replace $q-1$ with $s$ in above relation, then we find

$$
\int_{0}^{1} \frac{1-t^{s}}{1-t} d t=\psi(s+1)+\gamma
$$

Hence

$$
\psi(s+1)=-\gamma+\int_{0}^{1} \frac{1-t^{s}}{1-t} d t
$$


Theorem 5.2.5 [1] Assume $s>0$, then

$$
\int_{0}^{\infty}\left(e^{-x}-(1+x)^{-s}\right) \frac{d x}{x}=\psi(s)
$$

Proof. Firstly, we use the following integral representations,

$$
\begin{aligned}
\int_{0}^{\infty} \int_{1}^{n} e^{-x s} d a d s & =\int_{0}^{\infty}\left[-\frac{1}{s} e^{-x s}\right]_{1}^{n} d s \\
& =\int_{0}^{\infty}\left[-\frac{1}{s} e^{-n s}+\frac{1}{s} e^{-s}\right] d s \\
& =\int_{0}^{\infty} \frac{e^{s}-e^{-n s}}{s} d s
\end{aligned}
$$

and

$$
\int_{1}^{n} \int_{0}^{\infty} e^{-x s} d s d a=\int_{1}^{n}\left[-\frac{1}{x} e^{-x s}\right]_{0}^{\infty} d x=\int_{1}^{n} \frac{d x}{x}=\ln n-\ln 1=\ln n
$$

This means that,

$$
\int_{0}^{\infty} \frac{e^{-s}-e^{-n s}}{s} d s=\ln n
$$

Secondly, take the logarithmic derivative of $\Gamma(a)=\int_{0}^{\infty} n^{a-1} e^{-n} d n$ and use the definition of digamma function (5.1.1), we find

$$
\Gamma^{\prime}(a)=\int_{0}^{\infty} n^{a-1} e^{-n}(\ln n) d n
$$

Use (5.2.6), then

$$
\begin{aligned}
\Gamma^{\prime}(a) & =\int_{0}^{\infty} n^{a-1} e^{-n} \int_{0}^{\infty} \frac{e^{-s}-e^{-n s}}{s} d s d n \\
& =\int_{0}^{\infty} n^{a-1} e^{-n}\left(\int_{0}^{\infty} \frac{e^{-s}}{s} d s-\int_{0}^{\infty} \frac{e^{-n s}}{s} d s\right) d n
\end{aligned}
$$




$$
=\int_{0}^{\infty}\left[e^{-s} \int_{0}^{\infty} n^{a-1} e^{-n} d n-\int_{0}^{\infty} n^{a-1} e^{-n(1+s)} d n\right] \frac{d s}{s}
$$

The transformation $n(1+s)=x$ in $(5.2 .7)$ gives

$$
\Gamma^{\prime}(a)=\Gamma(a) \int_{0}^{\infty}\left[e^{-x}-(1+x)^{-a}\right] \frac{d x}{x} .
$$

Hence

$$
\psi(a)=\frac{\Gamma^{\prime}(a)}{\Gamma(a)}=\int_{0}^{\infty}\left(e^{-x}-(1+x)^{-a}\right) \frac{d x}{x} .
$$

Theorem 5.2.6 [1] For $\operatorname{Re}(s)>0$,

$$
\psi(s)=\int_{0}^{\infty}\left(\frac{e^{-x}}{x}-\frac{e^{-x s}}{1-e^{-x}}\right) d x \quad(\operatorname{Re}(s)>0)
$$

Proof. If $\operatorname{Re}(p)>0$, we have

$$
\frac{1}{p}=\int_{0}^{\infty} e^{-p x} d x
$$

integrating both sides,with respect to $p$ from $p=1$ to $p=m$ and use Fubini's theorem [4], we find

$$
\begin{gathered}
\ln m=\int_{0}^{\infty} \int_{1}^{m} e^{-p x} d p d x \\
\ln m=\int_{0}^{\infty}\left[-\left.\frac{1}{x} e^{-p x}\right|_{1} ^{m}\right] d x
\end{gathered}
$$




$$
\ln m=\int_{0}^{\infty}\left[\frac{e^{-x}-e^{-m x}}{x}\right] d x
$$

Substitution (5.2.9) and (5.2.10) in (5.2.1), we find

$$
\psi(s)=\lim _{m \rightarrow \infty}\left\{\int_{0}^{\infty}\left(e^{-x}-e^{-m x}\right) \frac{d x}{x}-\sum_{n=0}^{m} \int_{0}^{\infty} e^{-(s+n) x} d x\right\}
$$

Using equation (3) of section (3.4) in [3] then, we get

$$
\psi(s)=\lim _{m \rightarrow \infty}\left\{\int_{0}^{\infty}\left(e^{-x}-e^{-m x}\right) \frac{d x}{x}-\int_{0}^{\infty} \frac{e^{-s x}\left(1-e^{-x(m+1)}\right)}{1-e^{-x}} d x\right\}
$$

In (5.2.11), $e^{-m t}$ and $e^{-x(m+1)}$ approaches zero when $m$ goes to $\infty$. Thus

$$
\psi(s)=\int_{0}^{\infty}\left\{\frac{e^{-x}}{x}-\frac{e^{-s x}}{1-e^{-x}}\right\} d x
$$

\subsection{Generalization of the Psi (Digamma) Function}

In [1], M. Aslam Chaudhry and Syed M. Zubair was defined generalization of the Psi function as the following,

$$
\begin{gathered}
\psi_{c}(s)=\frac{d}{d s}\left\{\ln \left(\Gamma_{c}(s)\right)\right\}=\frac{1}{\Gamma_{c}(s)} \frac{d}{d s}\left\{\Gamma_{c}(s)\right\} \\
=\frac{1}{\Gamma_{c}(s)} \int_{0}^{\infty} t^{s-1} \ln t e^{-t-\frac{c}{t}} d t
\end{gathered}
$$




\subsection{Integral Representation of $\psi_{c}(s)$}

Theorem 5.4.1 [1]For $\operatorname{Re}(c) \geq 0$ or $c=0$ and $\operatorname{Re}(s)>0$,

$$
\psi_{c}(s)=\int_{0}^{\infty}\left\{e^{-a}-(1+a)^{-s} \frac{\Gamma_{c(1+a)}(s)}{\Gamma_{c}(s)}\right\} \frac{d a}{a} .
$$

Proof. Let us consider the following integral

$$
I=\int_{0}^{\infty} t^{s-1} \ln t e^{-t-\frac{c}{t}} d t
$$

substitution integral representation of $\ln t$ in (5.4.2) yields

$$
I=\int_{0}^{\infty} \int_{0}^{\infty} t^{s-1} e^{-\frac{c}{t}}\left\{\frac{e^{-t-a}-e^{-t(1+a)}}{a}\right\} d t d a .
$$

If we integrate the double integral with respect to $t$ we find

$$
I=\int_{0}^{\infty}\left\{e^{-a} \int_{0}^{\infty} t^{s-1} e^{-t-\frac{c}{t}} d t-\int_{0}^{\infty} t^{s-1} e^{-t(1+a)-c t^{-1}} d t\right\} \frac{d a}{a}
$$

From, (4.1.5) and $\Gamma_{c}(s)(4.1 .1)$, we find

$$
I=\int_{0}^{\infty}\left\{e^{-a} \Gamma_{c}(s)-\frac{1}{(1+a)^{s}} \Gamma_{c(1+a)}(s)\right\} \frac{d a}{a}
$$

Now, if we the integrate double integral (5.4.3) with respect to $a$, we find

$$
I=\int_{0}^{\infty} t^{s-1} e^{-t-\frac{c}{t}}\left\{\int_{0}^{\infty} \frac{e^{-a}-e^{-t a}}{a} d a\right\} d t
$$


Use the integral representation of $\ln t$ (5.2.10), we find

$$
\begin{aligned}
I & =\int_{0}^{\infty} t^{s-1} e^{-t-\frac{c}{t}} \ln t d t \\
& =\frac{d}{d s}\left\{\int_{0}^{\infty} t^{s-1} e^{-t-\frac{c}{t}} d t\right\} \\
& =\frac{d}{d s}\left(\Gamma_{c}(s)\right) .
\end{aligned}
$$

From (5.4.4) and (5.4.5), we get

$$
\frac{d}{d s}\left(\Gamma_{c}(s)\right)=\int_{0}^{\infty}\left\{e^{-a} \Gamma_{c}(s)-(1+a) \Gamma_{c(1+a)}(s)\right\} \frac{d a}{a}
$$

If we multiply the both sides in (5.4.6) by $\frac{1}{\Gamma_{c}(s)}$, we find

$$
\frac{d}{d s}\left(\Gamma_{c}(s)\right)=\int_{0}^{\infty}\left\{e^{-a} \frac{\Gamma_{c}(s)}{\Gamma_{c}(s)}-(1+a)^{s} \frac{\Gamma_{c(1+a)}(s)}{\Gamma_{c}(s)}\right\} \frac{d a}{a}
$$

Hence,

$$
\psi_{c}(s)=\left\{e^{-a}-(1+a)^{-s} \frac{\Gamma_{c(1+a)}(s)}{\Gamma_{c}(s)}\right\} \frac{d a}{a}
$$

Corollary 5.4.2 [1] (Dirichlet) For $\operatorname{Re}(s)>0$,

$$
\psi(s)=\int_{0}^{\infty}\left(e^{-a}-\frac{1}{(1+a)^{s}}\right) \frac{d a}{a} .
$$


Proof. The case $c=0,(5.4 .1)$ produces

$$
\psi_{0}(s)=\int_{0}^{\infty}\left\{e^{-a}-(1+a)^{-s} \frac{\Gamma_{0(1+a)}(s)}{\Gamma_{0}(s)}\right\} \frac{d a}{a}
$$

Using (5.4.1), we find

$$
\psi(s)=\int_{0}^{\infty}\left(\frac{e^{-a}-(1+a)^{-s}}{a}\right) d a
$$

Theorem 5.4.3 [1] For $\operatorname{Re}(c) \geq 0$ or $c=0$ and $\operatorname{Re}(s)>0$,

$$
\psi_{c}(s)=\int_{0}^{\infty}\left(\frac{e^{-x}}{x}-\frac{\Gamma_{c e^{x}}(s)}{\Gamma_{c}(s)} \frac{e^{-s x}}{1-e^{-x}}\right) d x
$$

Proof. From (5.4.1), we find

$$
\psi_{c}(s)=\lim _{\delta \rightarrow 0}\left[\int_{0}^{\infty} \frac{e^{-a}}{a} d a-\int_{\delta}^{\infty} \frac{1}{(1+a)^{s} a} \frac{\Gamma_{c(1+a)}(s)}{\Gamma_{c}(s)} d a\right]
$$

The change of variable $e^{x}=a+1$ in the second integral yields

$$
\begin{gathered}
\int_{\delta}^{\infty} \frac{(1+a)^{-s}}{a} \frac{\Gamma_{c(1+a)}(s)}{\Gamma_{c}(s)} d a=\int_{\ln (\delta+1)}^{\infty}\left\{\frac{e^{-x s}}{e^{-x}\left(e^{x}-1\right)} \frac{\Gamma_{c e^{x}}}{\Gamma_{c}(s)}\right\} d t \\
=\int_{\ln (\delta+1)}^{\infty}\left\{\frac{e^{-x s}}{1-e^{-x}} \frac{\Gamma_{c e^{x}}}{\Gamma_{c}(s)}\right\} d t
\end{gathered}
$$


From (5.4.8) and (5.4.9), we find

$$
\begin{aligned}
\psi_{c}(s) & =\lim _{\delta \rightarrow 0^{+}}\left[\int_{\delta}^{\ln (\delta+1)} \frac{e^{-a}}{a} d a+\lim _{\delta \rightarrow 0} \int_{\ln (\delta+1)}^{\infty}\left\{\frac{e^{-x}}{x}-\frac{e^{-x s}}{1-e^{-x}} \frac{\Gamma_{c e^{x}}}{\Gamma_{c}(s)}\right\} d x\right] \\
& =\int_{0}^{\infty}\left(\frac{e^{-x}}{x}-\frac{\Gamma_{c e^{x}}(s)}{\Gamma_{c}(s)} \frac{e^{-x s}}{1-e^{-x}}\right) d x
\end{aligned}
$$

Since

$$
\begin{aligned}
& \left|\int_{\delta}^{\ln (\delta+1)} \frac{e^{-x}}{x} d x\right| \leq \int_{\ln (\delta+1)}^{\delta} \frac{1}{x} d x \\
& =\ln t \mid \begin{array}{l}
\delta \\
\ln (\delta+1)
\end{array} \\
& =\ln \delta-\ln (\ln (\delta+1)) \\
& =\ln \left[\frac{\delta}{\ln (\delta+1)}\right] \rightarrow 0,\left(\text { as } \delta \rightarrow 0^{+}\right) .
\end{aligned}
$$

Corollary 5.4.4 [1] (Gauss) For $\operatorname{Re}(s)>0$,

$$
\psi(s)=\int_{0}^{\infty}\left\{\frac{e^{-x}}{x}-\frac{e^{-x s}}{1-e^{-x}}\right\} d x
$$

Proof. The special case $c=0$ in (5.4.7) produces

$$
\begin{aligned}
\psi_{c}(s)=\psi_{0}(s) & =\int_{0}^{\infty}\left\{\frac{e^{-x}}{x}-\frac{\Gamma_{0 e^{x}}}{\Gamma_{0}(s)} \frac{e^{-x s}}{1-e^{-x}}\right\} d x \\
& =\int_{0}^{\infty}\left\{\frac{e^{-x}}{x}-\frac{e^{-x s}}{1-e^{-x}}\right\} d x
\end{aligned}
$$


Theorem 5.4.5 [1] For $\operatorname{Re}(c) \geq 0$ and $\operatorname{Re}(s)>0$

$$
\psi_{c}(s)=\ln s+\int_{0}^{\infty}\left\{\frac{1}{x}-\frac{1}{1-e^{-x}} \frac{\Gamma_{c e^{x}}}{\Gamma_{c}(s)}\right\} e^{-s x} d x
$$

Proof. Adding and substraction $\frac{1}{x e^{s x}}$ in the first part of the integral in (5.4.7), we find

$$
\begin{aligned}
\psi_{c}(s) & =\int_{0}^{\infty}\left\{\frac{e^{-x}}{x}-\frac{e^{-s x}}{x}+\frac{e^{-s x}}{x}-\frac{e^{-s x}}{1-e^{-x}} \frac{\Gamma_{c e^{x}}}{\Gamma_{c}(s)}\right\} d x \\
& =\int_{0}^{\infty} \frac{e^{-x}-e^{-s x}}{x} d x+\int_{0}^{\infty}\left\{\frac{1}{x}-\frac{1}{1-e^{-x}} \frac{\Gamma_{c e^{x}}}{\Gamma_{c}(s)}\right\} e^{-s x} d x
\end{aligned}
$$

First integral is a integral representation of $\ln s$. Hence,

$$
\psi_{c}(s)=\ln s+\int_{0}^{\infty}\left\{\frac{1}{x}-\frac{1}{1-e^{-x}} \frac{\Gamma_{c e^{x}}}{\Gamma_{c}(s)}\right\} e^{-s x} d t
$$

Corollary 5.4.6 [1] For $\operatorname{Re}(s)>0$,

$$
\psi(s)=\ln s+\int_{0}^{\infty}\left\{\frac{1}{x}-\frac{1}{1-e^{-x}}\right\} e^{-s x} d x
$$

Proof. The above expression is a special case of (5.4.11) when, we put $c=0$

$$
\psi_{0}(s)=\ln s+\int_{0}^{\infty}\left\{\frac{1}{x}-\frac{1}{1-e^{-x}} \frac{\Gamma_{0 e^{x}}}{\Gamma_{0}(s)}\right\} e^{-s x} d x
$$


Hence

$$
\begin{gathered}
=\ln s+\int_{0}^{\infty}\left\{\frac{1}{x}-\frac{1}{1-e^{-x}}\right\} e^{-s x} d x \\
\psi_{0}(s)=\psi_{c}(s)=\ln s+\int_{0}^{\infty}\left\{\frac{1}{x}-\frac{1}{1-e^{-x}}\right\} e^{-s x} d x
\end{gathered}
$$

Proposition 5.4.7 [12] For $\operatorname{Re}(s)>0$,

Proof. The change of variable $t=e^{-x}$ in (5.2.8) yields,

$$
-\psi(s)=\int_{0}^{1}\left(\frac{1}{\ln t}+\frac{t^{s-1}}{1-t}\right) d t
$$

Theorem 5.4.8 [1] For $\operatorname{Re}(c)>0$ or $c=0$ and $\operatorname{Re}(s)>0$,

$$
\psi_{c}(s)=-\gamma+\int_{0}^{1}\left\{1-\left(\frac{\Gamma_{c t^{-1}}(s)}{\Gamma_{c}(s)}\right) t^{s-1}\right\} \frac{d t}{1-t} .
$$

Proof. The substitution $t=e^{-x}$ in (5.4.7) yields,

$$
\begin{aligned}
\psi_{c}(s) & =\int_{0}^{1}\left(\frac{t}{-\ln t}+\frac{\Gamma_{c t^{-1}}}{\Gamma_{c}(s)} \frac{t^{s}}{1-t}\right) \frac{-1}{t} d t \\
& =-\int_{0}^{1}\left(\frac{1}{\ln t}+\frac{\Gamma_{c t^{-1}}}{\Gamma_{c}(s)} \frac{t^{s-1}}{1-t}\right) d t .
\end{aligned}
$$


Adding and subtracting $\frac{1}{1-t}$ in the integrand (5.4.14), we find

$$
\begin{aligned}
& \psi_{c}(s)=-\left[\int_{0}^{1} \frac{1}{\ln t}+\frac{1}{1-t}+\frac{\Gamma_{c t^{-1}}}{\Gamma_{c}(s)} \frac{t^{s-1}}{1-t}-\frac{1}{1-t}\right] d t \\
& =-\int_{0}^{1}\left(\frac{1}{\ln t}+\frac{1}{1-t}\right) d x+\int_{0}^{1}\left(1-\frac{\Gamma_{c t}-1}{\Gamma_{c}(s)} t^{s-1}\right) \frac{d t}{1-t}
\end{aligned}
$$

Hence, using proposition 5.4.7, we find

$$
\psi_{c}(s)=-\gamma+\int_{0}^{1}\left\{1-\left(\frac{\Gamma_{c t^{-1}}(t)}{\Gamma_{c}(s)}\right) t^{s-1}\right\} \frac{d t}{1-t}
$$

Corollary 5.4.9 For $\operatorname{Re}(\alpha)>0$

$$
\psi(s)=-\gamma \int_{0}^{1}\left(\frac{1-t^{s-1}}{1-t}\right) d t
$$

Proof. The $c=0$ in $(5.4 .13)$ produces

$$
\psi_{0}(s)=\psi_{c}(s)=-\gamma+\int_{0}^{1}\left\{1-t^{s-1}\right\} \frac{d t}{1-t}
$$

\subsection{Properties of the Generalized psi Function}

Theorem 5.5.1 [1] (Reflection formula) For $\operatorname{Re}(c)>0$,

$$
\psi_{c}(-s)=\ln c-\psi_{c}(s)
$$


Proof. Replace $s$ and $-s$ in (5.3.2), we find

$$
\psi_{c}(-s)=\frac{1}{\Gamma_{c}(-s)} \int_{0}^{\infty} t^{-s-1}(\ln t) e^{-t-\frac{c}{t}} d t
$$

The change of variable $t=c x^{-1}$ and $d t=-c x^{-2} d x$ in (5.5.2) yields

$$
\begin{aligned}
\psi_{c}(-s) & =\frac{1}{\Gamma_{c}(-s)} \int_{0}^{\infty}\left(c x^{-1}\right)^{-s-1} \ln \left(\frac{c}{x}\right) e^{-c x^{-1}-\frac{c}{c x^{-1}} c x^{-2} d x} \\
& =\frac{1}{\Gamma_{c}(-s)} \int_{0}^{\infty} c^{-\alpha} \frac{x^{\alpha+1}}{x^{2}} \ln \left(\frac{c}{x}\right) e^{-c x^{-1}-x} d x \\
& =\frac{c^{-s}}{\Gamma_{c}(-s)} \int_{0}^{\infty} x^{s-1}(\ln c-\ln x) e^{-c x^{-1}-x} d x
\end{aligned}
$$

Using the formula (5.4.12), we find

$$
\begin{aligned}
& \psi_{c}(-s)= \frac{1}{\Gamma_{c}(s)} \int_{0}^{\infty} x^{s-1}(\ln c-\ln x) e^{-c x^{-1}-x} d x \\
&= \frac{1}{\Gamma_{c}(s)} \int_{0}^{\infty} x^{s-1}(\ln c) e^{-c x^{-1}-x} d x-\frac{1}{\Gamma_{c}(s)} \int_{0}^{\infty} x^{s-1}(\ln x) e^{-c x^{-1}-x} d x \\
&= \ln c \frac{1}{\Gamma_{c}(s)} \int_{0}^{\infty} x^{s-1}(\ln c) e^{-c x^{-1}-x} d x-\psi_{c}(s) \\
& \psi_{c}(-s)=\ln c-\psi_{c}(s)
\end{aligned}
$$

Corollary 5.5.2 [1] For $\operatorname{Re}(c)>0$,

$$
\psi_{c}(0)=\ln \sqrt{c}
$$


Proof. The special value $s=0$ in Reflection Formula (5.5.1) produces

$$
\begin{gathered}
-\psi_{c}(0)=\ln c-\psi_{c}(0) \\
\ln c=2 \psi_{c}(0) \\
\frac{1}{2} \ln c=\psi_{c}(0)
\end{gathered}
$$

Hence

$$
\ln \sqrt{c}=\psi_{c}(0)
$$

Corollary 5.5.3 [1] For $\operatorname{Re}(c)>0$

$$
\int_{0}^{\infty}(\ln t)\left[t^{s}-\left(\frac{c}{t}\right)^{s}\right] e^{-t-\frac{c}{t}} \frac{d t}{t}=2 c^{\frac{s}{2}}(\ln c) K_{s} 2 \sqrt{c}
$$

Proof. Replace $s$ by $-s$ in (5.5.1), we find

$$
\psi_{c}(s)=\ln c-\psi_{c}(-s)
$$

Substitution integral representation of $\psi_{c}(s)$ in (5.5.2), we get

$$
\frac{1}{\Gamma_{c}(s)} \int_{0}^{\infty} t^{s-1}(\ln t) e^{-t-\frac{c}{t}} d t=\ln c-\frac{1}{\Gamma_{c}(-s)} \int_{0}^{\infty} t^{-s-1} \ln (t) e^{-t-\frac{c}{t}} d t
$$


Using the formula (4.2.10)

$$
\begin{gathered}
\frac{1}{\Gamma_{c}(s)} \int_{0}^{\infty} t^{s-1}(\ln t) e^{-t-\frac{c}{t}} d t=\ln c-\frac{c^{s}}{\Gamma_{c}(s)} \int_{0}^{\infty} t^{-s-1} \ln (t) e^{-t-\frac{c}{t}} d t \\
\int_{0}^{\infty} t^{s-1}(\ln t) e^{-t-\frac{c}{t}} d t+t^{-s-1} c^{s} \ln (t) e^{-t-\frac{c}{t}} d t=\Gamma_{c}(s) \ln c \\
\ln c \Gamma_{c}(s)=\int_{0}^{\infty}(\ln t) e^{-t-c t^{-1}}\left[\left(\frac{c}{t}\right)^{s}+t^{s}\right] \frac{d t}{t} \\
\int_{0}^{\infty}(\ln t) e^{-t-\frac{c}{t}}\left\{t^{s-1}+c^{s} t^{-s-1}\right\} \frac{d t}{t}=\Gamma_{c}(s) \ln c
\end{gathered}
$$

Substitution Macdonald representation of $\Gamma_{c}(s)$ in (5.5.3), we find

$$
\int_{0}^{\infty}(\ln t) t^{s}+\left(\frac{c}{t}\right)^{s} e^{-t-\frac{c}{t}} \frac{d t}{t}=2 c^{\frac{s}{2}}(\ln c) K_{s}(2 \sqrt{c})
$$




\section{Chapter 6}

\section{THE GENERALIZED INCOMPLETE GAMMA FUNCTIONS}

The definition of the acculamated curve of the gamma distribution one of the many application of the incomplete gamma function.

\subsection{The Incomplete Gamma Functions}

The (lower) incomplete gamma function defined as the following,

$$
\gamma(s, x)=\int_{0}^{x} t^{s-1} e^{-t} d t \quad(s=\sigma+i r ; \sigma>0, \quad|\arg (s)|<\pi),
$$

and the upper Incomplete Gamma Function is defined as

$$
\Gamma(s, x)=\int_{x}^{\infty} t^{s-1} e^{-t} d t \quad(|\arg (s)|<\pi)
$$

The lower and upper Incomplete Gamma Functions were first invastigated for $x \in \mathbb{R}$ by Legendre [19].

\subsection{Definition of the Generalized Incomplete Gamma Functions}

In[19], Chaudhry and Zubair introduced the definition of Generalized Incomplete Gamma functions as

$$
\Gamma(s, x ; c)=\int_{x}^{\infty} t^{s-1} e^{-t-\frac{c}{t}} d t
$$




$$
\gamma(s, x ; c)=\int_{0}^{x} t^{s-1} e^{-t-\frac{c}{t}} d t
$$

where $s, x$ are complex parameters and $c$ is a complex variable. For $c=0$, we get

$$
\begin{gathered}
\gamma(s, x ; 0)=\gamma(s, x) \\
\Gamma(s, x ; 0)=\Gamma(s, x)
\end{gathered}
$$

\subsection{Properties of the Incomplete Generalized Gamma Functions}

Theorem 6.3.1 [1] (Decomposition theorem) For $\operatorname{Re}(c) \geqslant 0$,

$$
\gamma(s, x ; c)+\Gamma(s, x ; c)=\Gamma_{c}(s)
$$

Proof. When we add lower and upper incomplete gamma functions, we get

$$
\gamma(s, x ; c)+\Gamma(s, x ; c)=\int_{0}^{x} t^{s-1} e^{-t-c t^{-1}} d t+\int_{x}^{\infty} t^{s-1} e^{-t-c t^{-1}} d t
$$

Hence

$$
\int_{0}^{\infty} t^{s-1} e^{-t-c t^{-1}} d t=\Gamma_{c}(s)
$$


Theorem 6.3.2 [1] (Mellin transform representation) For $\operatorname{Re}(c)>0$ and $0<u<1$

$$
\Gamma(s, x ; c)=\frac{1}{2 \pi i} \int_{u-i \infty}^{u+i \infty} \Gamma(\alpha) \Gamma(s+\alpha, x) c^{-\alpha} d s
$$

Proof. Multiplying (6.2.1) by $c^{\alpha-1}$ gives

$$
\int_{0}^{\infty} c^{\alpha-1} \Gamma(s, x ; c) d c=c^{\alpha-1} \int_{x}^{\infty} t^{s-1} e^{-t-\frac{c}{t}} d t
$$

Integrating both sides with respect to $c$ from $c=0$ to $c=\infty$, we find

$$
\int_{0}^{\infty} c^{\alpha-1} \Gamma(s, x ; c) d c=\int_{0}^{\infty} c^{\alpha-1} \int_{x}^{\infty} t^{s-1} e^{-t-\frac{c}{t}} d t d c
$$

Using the Fubini Theorem [4], then

$$
\int_{0}^{\infty} c^{\alpha-1} \Gamma(s, x ; c) d c=\int_{x}^{\infty} t^{s-1} e^{-t}\left(\int_{0}^{\infty} c^{\alpha-1} e^{-\frac{c}{t}} d c\right) d t
$$

Let $c=t u$, then we get

$$
\begin{aligned}
& =\int_{x}^{\infty} t^{s-1} e^{-t}\left(\int_{0}^{\infty}(t u)^{\alpha-1} e^{u} t d u\right) d t \\
& =\int_{x}^{\infty} t^{s-1} e^{-t}\left(\int_{0}^{\infty}(u)^{\alpha-1} t^{s} e^{u} d u\right) d t \\
& =\int_{x}^{\infty} t^{s+\alpha-1} e^{-t} \int_{0}^{\infty} u^{\alpha-1} e^{u} d u
\end{aligned}
$$

Second integral is a standart form of the Gamma Function

$$
\int_{0}^{\infty} c^{\alpha-1} \Gamma(s, x ; c) d c=\Gamma(\alpha) \int_{x}^{\infty} t^{s+\alpha-1} e^{-t} d t .
$$


So

$$
\int_{0}^{\infty} c^{\alpha-1} \Gamma(s, x ; c) d c=\Gamma(\alpha) \Gamma(s+\alpha, x)
$$

Use the inverse Mellin Transform defined as (2.0.4), we find

$$
\Gamma(s, x ; c)=\frac{1}{2 \pi i} \int_{u-i \infty}^{u+i \infty} c^{-\alpha} \Gamma(\alpha) \Gamma(s+\alpha, x) d s
$$

Theorem 6.3.3 [19]For $a>0$,

$$
\int_{x}^{\infty} t^{s-1} e^{-a t-c t^{-1}} d t=a^{-s} \Gamma(s, a x ; a c)
$$

Proof. Substitution $t=\frac{\mu}{a}$ in $\int_{x}^{\infty} t^{s-1} e^{-a t-c t^{-1}} d t$ and use (6.2.1), we get

$$
a^{-s} \int_{a x}^{\infty}(\mu)^{s-1} e^{-\mu-a c \mu^{-1}} d \mu=a^{-s} \Gamma(s, a x ; a b)
$$

Theorem 6.3.4 [19] (Reccurence Relation)

$$
\Gamma(s+1, x ; c)=s \Gamma(s, x ; c)+c \Gamma(s-1, x ; c)+x^{s} e^{-x-c x^{-1}}
$$

\section{Proof.}

$$
\frac{d}{d x}\left(x^{s} e^{-x-c x^{-1}}\right)=\left(s x^{s-1}+c x^{\alpha-2}-x^{s}\right) e^{-x-c x^{-1}} .
$$


By the definition of Generalized Incomplete Gamma Function as (6.2.1) and use the Fundamental Theorem [21] to integrate both sides in (6.3.6), we find

$$
\begin{aligned}
& \frac{d}{d x}\left(\int_{x}^{\infty} t^{s} e^{-t-c t^{-1}} d t\right)=\left[\infty^{s} \frac{1}{e^{\infty+c \frac{1}{\infty}}}-x^{s} e^{-x-c x^{-1}}\right] \\
&=-x^{s} e^{-x-c x^{-1}} \\
&-x^{s} e^{-x-c x^{-1}}=s \Gamma(s, x ; c)+c \Gamma(s-1, x ; c)-\Gamma(s+1, x ; c)
\end{aligned}
$$

which is exactly $(6.3 .5)$.

Corollary 6.3.5 [19]

$$
\Gamma(s+1, x)=s \Gamma(s, x)+x^{s} e^{-x}
$$

Proof. For $c=0$ in $(6.3 .5)$, produces

$$
\Gamma(\alpha+1, x ; 0)=s \Gamma(s, x ; 0)+c \Gamma(s-1, x ; 0)+e^{-x-c x^{-1}}
$$

Use (6.2.4), then

$$
\Gamma(s+1, x)=s \Gamma(s, x)+x^{s} e^{-x}
$$

which is exactly $(6.3 .7)$.

Theorem 6.3.6 [19] (Differentiation formula)

$$
\frac{d}{d x}(\Gamma(s, x ; c))=-x^{s-1} e^{-x-c x^{-1}}
$$


Proof. Integrating both sides with respect to $x$ from (6.2.1) and use fundamental Theorem [21], then

$$
\begin{aligned}
\frac{d}{d x}(\Gamma(s, x ; c)) & =\frac{d}{d x}\left(\int_{x}^{\infty} t^{s-1} e^{-t-c t^{-1}} d t\right) \\
& =\left[\infty^{s-1} \frac{1}{e^{\infty+c \frac{1}{\infty}}}-x^{s-1} e^{-x-c x^{-1}}\right] \\
& =-x^{s-1} e^{-x-c x^{-1}}
\end{aligned}
$$

This concludes the proof.

Corollary 6.3.7 [19]

$$
\frac{d}{d x}(\Gamma(s+1, x ; c))=-x^{s-1} e^{-x}
$$

Proof. For $c=0$ in $(6.3 .8)$, produces

$$
\begin{aligned}
\frac{d}{d x}(\Gamma(s, x ; c)) & =\frac{d}{d x}\left(\int_{x}^{\infty} t^{s-1} e^{-t-c t^{-1}} d t\right) \\
\frac{d}{d x}(\Gamma(s, x ; 0)) & =\left[\infty^{s-1} \frac{1}{e^{\infty+0 \frac{1}{\infty}}}-x^{s-1} e^{-x-0 x^{-1}}\right] \\
& =-x^{s-1} e^{-x}
\end{aligned}
$$

This concludes the proof. 


\section{Chapter 7}

\section{EXTENDED BETA FUNCTION}

This chapter contains basic definitions and properties of the extended beta functions.

\subsection{Definition of the Extended Beta Function}

Definition 7.1.1 [1]For $\operatorname{Re}(c)>0, y$ and $x$ arbitary complex number the Extended Beta Function is defined as

$$
B(x, y ; c)=\int_{0}^{1} t^{x-1}(1-t)^{y-1} e^{-\frac{c}{t(1-t)}} d t
$$

For $c=0, \operatorname{Re}(x)>0$ and $\operatorname{Re}(y)>0$, we get Ordinary Beta Function.

\subsection{Properties of the Extended Beta Functions}

Theorem 7.2.1 [1]For $\operatorname{Re}(c) \geq 0$,

$$
B(x, y ; c)=B(y, x ; c) .
$$

Proof. Replace $t$ by $1-t$ in (7.2.1), we find

$$
\int_{0}^{1}(1-t)^{x-1} t^{y-1} e^{-\frac{c}{t(1-t)}} d t=B(y, x ; c)
$$

Theorem 7.2.2 [1] (Functional relation)

$$
B(x, y+1 ; c)+B(x+1, y ; c)=B(x, y ; c)
$$


Proof. Using the integral representations of the Extended Beta function [13], we find

$$
\begin{aligned}
B(x, y+1 ; c)+B(x+1, y ; c) & =\int_{0}^{1} t^{x-1}(1-t)^{y} e^{-\frac{c}{t(1-t)}} d t+\int_{0}^{1} t^{x}(1-t)^{y-1} e^{-\frac{c}{t(1-t)}} d t \\
& =\int_{0}^{1} t^{x}(1-t)^{y} e^{-\frac{c}{t(1-t)}}\left[\frac{1}{t(1-t)}\right] d t \\
& =\int_{0}^{1} t^{x-1}(1-t)^{y-1} e^{-\frac{c}{t(1-t)}} d t
\end{aligned}
$$

which is exactly same in (7.2.2).

Theorem 7.2.3 [1] (Infinite sum) For $\operatorname{Re}(c)>0$,

$$
B(x, y ; c)=\sum_{n=0}^{\infty} B(x+n, y+1 ; c) \quad(\operatorname{Re}(c)>0)
$$

Proof. The factor $(1-t)^{y-1}$ has the series representations as the following

$$
(1-t)^{y-1}=(1-t)^{y} \sum_{n=0}^{\infty} t^{n}
$$

So,

$$
\begin{aligned}
B(x, y ; c) & =\int_{0}^{1} t^{x-1}(1-t)^{y} \sum_{n=0}^{\infty} t^{n} e^{-\frac{c}{t(1-t)}} d t \\
& =\sum_{n=0}^{\infty} \int_{0}^{1}(1-t)^{y} t^{x+n-1} e^{-\frac{c}{t(1-t)}} d t \\
& =B(x+n, y+1 ; c)
\end{aligned}
$$

This concludes the proof. 
Theorem 7.2.4 [1] (Infinite sum) For $\operatorname{Re}(c)>0$,

$$
B(x, 1-y ; c)=\sum_{n=0}^{\infty} \frac{(y)_{n}}{n !} B(x+n, 1 ; c)
$$

Proof. Using the definition of extended beta function [15], we get

$$
B(x, 1-y ; c)=\int_{0}^{1} t^{x-1}(1-t)^{-y} e^{-\frac{c}{t(1-t)}} d t
$$

The factor $(1-t)^{-y}$ has the series representations as the following

$$
(1-t)^{-y}=\sum_{n=0}^{\infty}(y)_{n} \frac{t^{n}}{n !}
$$

using (7.2.7) in (7.1.1), we obtain

$$
B(x, 1-y ; c)=\int_{0}^{1} \sum_{n=0}^{\infty} \frac{(y)_{n}}{n !} t^{x+n-1} e^{-\frac{c}{t(1-t)}} d t .
$$

For $\operatorname{Re}(b)>0$, the order of integration and summation is change

$$
\begin{aligned}
B(x, 1-y ; c) & =\sum_{n=0}^{\infty} \frac{(y)_{n}}{n !} \int_{0}^{1} t^{x+n-1} e^{-\frac{c}{t(1-t)}} d t \\
& =\sum_{n=0}^{\infty} \frac{(y)_{n}}{n !} B(x+n, 1 ; c)
\end{aligned}
$$

This concludes the proof.

Theorem 7.2.5 [1] (An inequality) For $p>0, q>0$ and $c \geqslant 0$, then

$$
B(p, q ; c) \leq \exp (-4 c) B(p, q)
$$


Proof. The transformation $t=\frac{u}{1+u}$ and $d t=\frac{1}{1+u}-\frac{u}{(1+u)^{2}} d u$ in the integral representation of the Extented Beta Function (7.1.1), yields

$$
\begin{aligned}
B(p, q ; c) & =\int_{0}^{\infty}\left[\left(\frac{u}{1+u}\right)^{p-1}\left(\frac{1}{1+u}\right)^{q-1} \exp \left(-c \frac{(1+u)^{2}}{u}\right)\left(\frac{1}{1+u}-\frac{u}{(1+u)^{2}}\right)\right] d u \\
& =\int_{0}^{\infty} u^{p-1}(1+u)^{1-p}(1+u)^{1-q} \frac{1}{(1+u)^{2}} \exp \left[-c \frac{\left(1+2 u+u^{2}\right)}{u}\right] d u \\
& =\int_{0}^{\infty} u^{p-1}(1+u)^{-p}(1+u)^{-q} \exp \left[-c\left(1+2 u+u^{2}\right) u^{-1}\right] d u \\
& =\int_{0}^{\infty} u^{p-1} \frac{1}{(1+u)^{p+q}} \exp \left[-c\left(u^{-1}+2+u\right)\right] d u
\end{aligned}
$$

so,

$$
B(p, q ; c)=\exp (-2 c) \int_{0}^{\infty} \frac{u^{p-1}}{(1+u)^{p+q}} \exp \left[-c\left(u^{-1}+u\right)\right] d u
$$

For $u=1, \exp \left[-c\left(u^{-1}+u\right)\right]$ takes the mean value. Hence

$$
B(p, q ; c) \leq \exp (-4 c) \frac{u^{p-1}}{(1+u)^{p+q}} d u
$$

which is exactly (7.2.8).

Next section contain same integral representations of extended beta function.

\subsection{Integral Representations of the Extended Beta Function}

Theorem 7.3.1 [1]

$$
B(x, y ; c)=2 \int_{0}^{\frac{\pi}{2}}(\cos \theta)^{2 x-1}(\sin \theta)^{2 y-1} e^{-c \sec ^{2} \theta \csc ^{2} \theta} d \theta
$$


Proof. The transformation $t=\sin ^{2} \theta$ in the definition of the Extended Beta Function yields

$$
\begin{aligned}
B(x, y ; c) & =2 \int_{0}^{\frac{\pi}{2}}\left[\left(\sin ^{2} \theta\right)^{x-1}\left(1-\sin ^{2} \theta\right)^{y-1} e^{\frac{-c}{\sin ^{2} \theta\left(1-\sin ^{2} \theta\right)}} \cos \theta \sin \theta\right] d \theta \\
& =2 \int_{0}^{\frac{\pi}{2}}(\sin \theta)^{2 x-2}(\cos \theta)^{2 y-2} e^{\frac{-c}{\sin ^{2} \theta \cos ^{2} \theta} \cos \theta \sin \theta d \theta} \\
& =2 \int_{0}^{\frac{\pi}{2}}(\sin \theta)^{2 x-1}(\cos \theta)^{2 y-1} e^{-c \frac{1}{\sin ^{2} \theta} \frac{1}{\cos ^{2} \theta} d \theta} \\
& =2 \int_{0}^{\frac{\pi}{2}}(\sin \theta)^{2 x-1}(\cos \theta)^{2 y-1} e^{-c \csc ^{2} \theta \sec ^{2} \theta} d \theta
\end{aligned}
$$

which is exactly same in $(7.3 .1)$.

Theorem 7.3.2 [1]

$$
B(x, y ; c)=e^{-2 c} \int_{0}^{\infty} \frac{u^{x-1}}{(1+u)^{x+y}} \exp \left[-c\left(u^{-1}+u\right)\right] d u
$$

Proof. The trasformation $t=\frac{u}{1+u}$ and $d t=\frac{1}{1+u}-\frac{u}{(1+u)^{2}} d u$ in the integral representation of the Extented Beta Function (7.1.1), yield

$$
\begin{aligned}
B(x, y ; c) & =\int_{0}^{\infty}\left[\left(\frac{u}{1+u}\right)^{x-1}\left(\frac{1}{1+u}\right)^{y-1} \exp \left(-c \frac{(1+u)^{2}}{u}\right)\left(\frac{1}{1+u}-\frac{u}{(1+u)^{2}}\right)\right] d u \\
& =\int_{0}^{\infty} \frac{u^{x-1}}{(1+u)^{x-1}} \frac{1}{(1+u)^{y-1}} \exp \left(-c \frac{(1+u)^{2}}{u}\right) \frac{1}{(1+u)^{2}} d u \\
& =\int_{0}^{\infty} \frac{u^{x-1}}{(1+u)^{x}} \frac{1}{(1+u)^{y}} \exp \left[-c\left(\frac{1+2 u+u^{2}}{u}\right)\right] d u \\
& =e^{-2 c} \int_{0}^{\infty} \frac{u^{x-1}}{(1+u)^{x+y}} \exp \left[-c\left(u^{-1}+u\right)\right] d u .
\end{aligned}
$$


Theorem 7.3.3 [1]

$$
B(x, y ; c)=2^{1-x-y} \int_{-1}^{1}(1+t)^{x-1}(1-t)^{y-1} \exp \left[\frac{-4 c}{1-t^{2}}\right] d u
$$

Proof. The trasformation $t=\frac{u-a}{d-a}$ in the integral representation of the Extended Beta Function (7.1.1), yield

$$
\begin{aligned}
B(x, y ; c) & =\int_{a}^{c}\left[\left(\frac{u-a}{d-a}\right)^{x-1}\left(1-\frac{u-a}{d-a}\right)^{y-1} e^{\left[\frac{-c}{\frac{u-a}{d-a}\left(1-\frac{u-a}{d-a}\right)}\right]} \frac{1}{d-a}\right] d u \\
& =\int_{a}^{c}(u-a)^{x-1}(d-a)^{1-x}(d-u)^{y-1}(d-a)^{1-y}(d-a)^{-1} e^{\left[\frac{-c(d-a)^{2}}{(u-a)(d-u)}\right]} d u \\
& =(d-a)^{1-x-y} \int_{a}^{c}(u-a)^{x-1}(d-u)^{y-1} e^{\left[\frac{-c(d-a)^{2}}{(u-a)(d-u)}\right]} d u
\end{aligned}
$$

This is a special case of (7.3.3) when we take $a=-1$ and $d=1$,

$$
B(x, y ; c)=2^{1-x-y} \int_{-1}^{1}(1+u)^{x-1}(1-u)^{y-1} e^{\left[\frac{-4 c}{1-u^{2}}\right]} d u
$$

which is exactly (7.3.4). 


\section{REFERENCES}

[1] M.Aslam Chaudhry, Syed M. Zubair, On a Class of Incomplete Gamma Functions with Application, Chapman and Hall / CRC 2002

[2] Dimitru Baleanu ,Kai Diethelm, Enrico Scalas, Juan J.Trujillo Fractional Calculus: Models and Numerical Methods, World Scientific 2012

[3] Leon M.Hall, Special Functions, Professor of Mathematics, University of Missouri-Rolla 1995

[4] Budak,B.M and Fomin, S.V.Multiple Integrals, Field Theory and Series (Translated from Russon by V.M Volosov), Mir Publisleis ,Moscow 1978

[5] L. S. Gradshteyn and L. M. Ryzhink. Tables of Integrals,Series and Products. Edided by A. Jeffrey and D. Zwissinger. Academic Press, Newyork, 7th edition, 2007

[6] Abramowitz,M and I. A. Stegun, 1972. Modified Bessel Function L and K, Handbook of Mathematical Functions with Formulas, Graphs and Mathematical Tables, Newyork

[7] S.Karris,Mathematics for Business,Science and Technology,Orchard Publications, 2007

[8] K.Kon, Fractional-Calculus.com, Allien's Mathematics 2003 
[9] Z.X.Wang, D.R.Guo, Special Function, World Scientific 1989

[10] Wikipedia. An online resource. http://en.wikipedia.org/wiki

[11] Det Kgl.Danske Videnskabernes Selskab, Kobenhavn, Hovedkommissioner : Andr.Fred. Host and Son,Kgl.Holf-Boghandel, On the Logarithmic Derivatives of the Gamma Function by Einar Hille, Mathematisk-fysiske Meddeleser, Bianco Lunos Borgtrykkeri 1927

[12] Luis A. Medina and Victor H. Moll, The integrals in Gradshteyn and Ryzhik. Part 10: The Digamma Function, Series A:Mathematical Sciences Vol. 17(2009), $45-66$

[13] Dong Myung Lee, Argun K. Rathie,Rakesh K. Parmar and Yong Sup Kim, Generazilation of Extended Beta Function, Hypergeometric and Confluent Hypergeomeric Functions, Honam Mathematical J.33(2011), No:2, pp.187-206

[14] G.E.Andrews, R.Askey and R.Roy, Special Functions,Encyclopedia of Mathematics and Hs Applications 71, Cambridge University Press, 1999

[15] M.Aslam Chaudhry, Asghar Qadir, M.Rafique,S. M. Zubair, Extension of Euler's Beta Function, Journal of Computational and Applied Mathematics 78 (1997), $19-32$

[16] P. Flajolet and M. Golin, Mellin Transforms and Asymptotics. Acta Information, (1994), 673-696 
[17] Wolfram, A wolfram web resource. http://functions.wolfram.com /GammaBetaErf/Gammal

[18] Pascal Sebah and Xaviere Gourdon, Introduction to the Gamma Function, numbers.computation.free.fr/Constants/constants.html, 2002

[19] M.A Chaudhry, S. M. Zubair, Generalized Incomplete Gamma Functions with Applications, J.Comput.App.Math 55, (1994), 99-124.

[20] M.Aslam Chaudhry, Asghar Qadir, Hypergeometric Function. App.Math Compact, 159, (2004) 589-602

[21] Adams, Roberst A., Calculus:a complete course, 1940, p.328 